\title{
Anandamide Hydrolysis in FAAH Reveals a Dual Strategy for Efficient Enzyme-Assisted Amide Bond Cleavage via Nitrogen Inversion
}

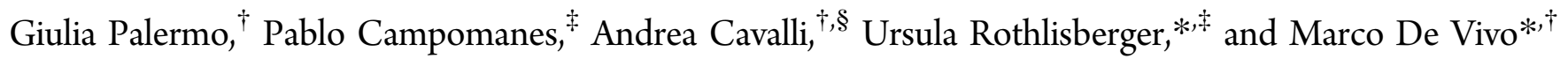 \\ ${ }^{\dagger}$ Department of Drug Discovery and Development, Istituto Italiano di Tecnologia, Via Morego 30, 16163 Genoa, Italy \\ ${ }^{\ddagger}$ Laboratory of Computational Chemistry and Biochemistry, Institute of Chemical Sciences and Engineering, Ecole Polytechnique \\ Fédérale de Lausanne, Lausanne (EPFL), CH-1015 Lausanne, Switzerland \\ ${ }^{\S}$ Department of Pharmacy and Biotechnology, University of Bologna, Via Belmeloro 6, I-40126 Bologna, Italy
}

\section{Supporting Information}

ABSTRACT: Herein, we combined classical molecular dynamics (MD) and quantum mechanical/molecular mechanics (QM/MM) simulations to unravel the whole catalytic cycle of fatty acid amide hydrolase (FAAH) in complex with anandamide, the main neurotransmitters involved in the control of pain. While microsecond MD simulations of FAAH in a realistic membrane/water environment provided a solid model for the reactant state of the enzymatic complex (Palermo et al. J. Chem. Theory Comput. 2013, 9, 1202-1213.), QM/MM simulations depict now a highly concerted two-step

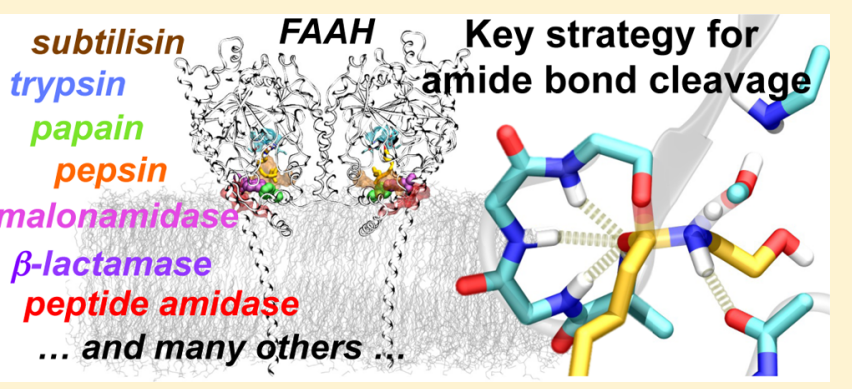
catalytic mechanism characterized by (1) acyl-enzyme formation after hydrolysis of the substrate amide bond and (2) deacylation reaction with restoration of the catalytic machinery. We found that a crucial event for anandamide hydrolysis is the inversion of the reactive nitrogen of the scissile amide bond, which occurs during the acylation rate-limiting step. We show that FAAH uses an exquisite catalytic strategy to induce amide bond distortion, reactive nitrogen inversion, and amide bond hydrolysis, promoting catalysis to completion. This new strategy is likely to be of general applicability to other amidases/peptidases that show similar catalytic site architectures, providing crucial insights for de novo enzyme design or drug discovery efforts.

\section{INTRODUCTION}

Endogenous cannabinoids are essential lipid-derived signaling molecules that activate $\mathrm{CB}_{1}$ cannabinoid receptors, and are involved in a variety of pathophysiological processes of the brain, endocrine, and immune systems. ${ }^{1,2}$ Anandamide ( $N$ arachidonoyl ethanolamine) was the first endocannabinoid to be discovered, and is one of the major players in the endocannabinoid-mediated control of pain., ${ }^{3,4}$ The biological actions of anandamide are primarily modulated by fatty acid amide hydrolase (FAAH; Figure 1), 5,6 an intracellular serine hydrolase that belongs to the amidase signature family. FAAH hydrolyzes anandamide in arachidonic acid and ethanolamine with a specific mechanism of degradation, which has been targeted by several drug discovery programs. Indeed, inhibition of FAAH enhances levels of endogenous cannabinoids, which is a promising strategy for the treatment of an ever-increasing number of pathologies, spanning from pain and inflammatoryrelated diseases such as cancer to neurological, psychiatric, and eating disorders. ${ }^{7-11}$

It has been shown that FAAH is a dimeric enzyme that can guide its substrates in and out of the catalytic site via a complex architecture, which has suggested an intriguing mechanism for substrate binding and product release (Figure 1). ${ }^{12}$ Substrates are thought to reach the catalytic site via a "membrane access" channel where two charged residues (Asp403 and Arg486) favor the entrance of the polar head groups of fatty acid substrates, while an adjacent "acyl-chain binding" cavity likely contributes to the accommodation of substrates during catalysis. ${ }^{13,14}$ Closely connected to the catalytic region, a solvent-exposed "cytosolic port" is appropriately located to grant the enzyme access to the cytosolic compartment of the cell. Indeed, the presence of the polar residues (Lys142 and Thr236) favors the leaving group departure toward the cytosol after substrate hydrolysis, followed by the entrance of water molecules that are needed for the turnover of the acyl-enzyme intermediate. ${ }^{1,13}$

Once the substrate is bound to FAAH, the catalytic reaction occurs in the core of its binding site where an unusual catalytic triad (Ser241-Ser217-Lys142) performs the hydrolysis of the substrate, while an oxyanion hole (Ser241-Gly240-Gly239Ile238) stabilizes the substrate for catalysis. Structural and kinetic data on FAAH catalysis have suggested a catalytic mechanism that involves a complex multievent reaction sequence. $^{12,15}$ In particular, the catalytic mechanism for

Special Issue: William L. Jorgensen Festschrift

Received: May 28, 2014

Revised: August 8, 2014

Published: September 10, 2014 


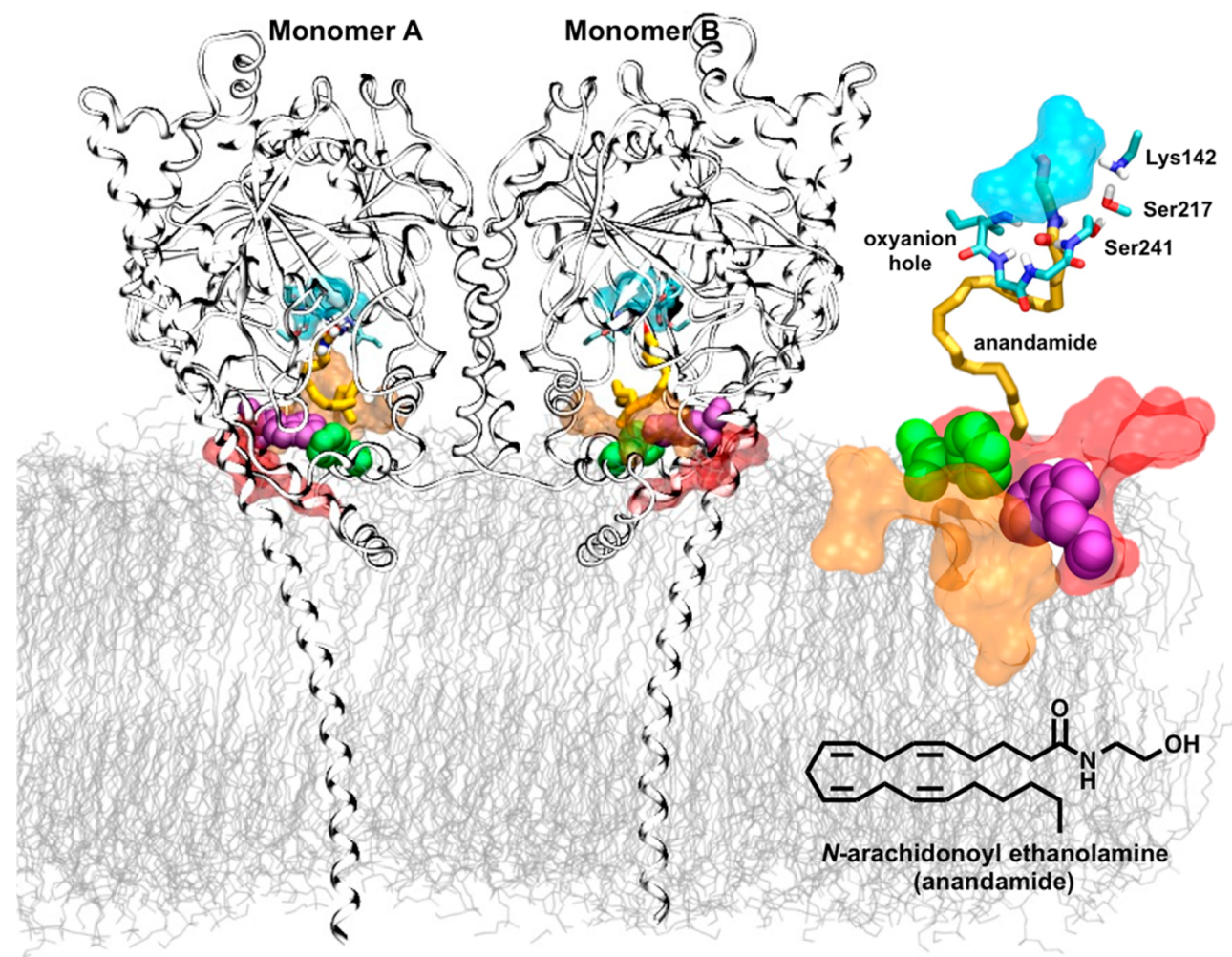

Figure 1. Overview of the simulated system of FAAH in complex with anandamide (chemical structure on the bottom right), embedded in a 1palmitoyl-2-oleoyl-phosphatidylethanolamine (POPE) lipid bilayer. The enzyme is a homodimer, formed by monomers A and B, which are depicted in gray ribbons. POPE lipids are shown in gray lines. The binding site of each FAAH monomer comprises three main (binding) channels (in molecular surface representation). ${ }^{12}$ Anandamide (shown in yellow sticks) is thought to reach the catalytic site via a "membrane access" channel (red molecular surface). An adjacent "acyl-chain binding" channel (orange) likely contributes to the accommodation of the substrate during catalysis. Key residues of the binding site (Phe432 and Trp531 in green and magenta space-filling representation) are also involved in favoring the substrate binding. At the top of the catalytic region, a third channel (cyan) constitutes the "cytosolic port" that allows the exit of the leaving group, after hydrolysis. ${ }^{13,14}$ The catalytic site is enlarged on the right, showing the anandamide substrate (yellow) surrounded by the catalytic triad (Ser241, Ser217, Lys142) and the oxyanion hole residues (Ser241, Gly240, Gly239, Ile238), shown as cyan sticks. For the sake of clarity, explicit water molecules included in the simulations are omitted.

anandamide hydrolysis comprises two main chemical steps: enzyme acylation (Step 1; $\mathrm{A} \rightarrow \mathrm{C}$ in Scheme 1) and enzyme deacylation (Step 2; D $\rightarrow$ F in Scheme 1). During the acylation reaction, the deprotonated Lys 142 activates the Ser241 nucleophile via proton transfers that involve Ser217 (A). The activated Ser241 attacks the anandamide carbonyl group, leading to the formation of a tetrahedral intermediate (B). At this point, a reversed proton transfer, from Lys 142 through Ser217, leads to the protonation of the leaving group (i.e., ethanolamine), thus obtaining the acyl-enzyme adduct (C). The deacylation reaction takes place upon exit of the ethanolamine leaving group from the catalytic site, most likely via the "cytosolic port" (Figure 1), followed by water molecules solvating the catalytic site and, hence, approaching the acylenzyme adduct. During the deacylation reaction, in fact, a water molecule acts as a nucleophilic agent, attacking the carbonyl group of the acyl-enzyme adduct (D; Scheme 1). A new proton transfer involving the catalytic triad allows the deprotonation of the nucleophilic water (E) to favor the formation and subsequent release of the final product. The restoration of the initial protonation state and ordered $\mathrm{H}$-bond network of the catalytic triad terminates the catalytic loop, with the catalyst FAAH ready for a new cycle $(F){ }^{13,16}$

The wealth of experimental data on FAAH catalysis and inhibition has made this enzyme a prototypical model system for investigating a complex multievent catalytic reaction
Scheme 1. Proposed Mechanism of Anandamide Hydrolysis Catalyzed by FAAH ${ }^{13,15 a}$

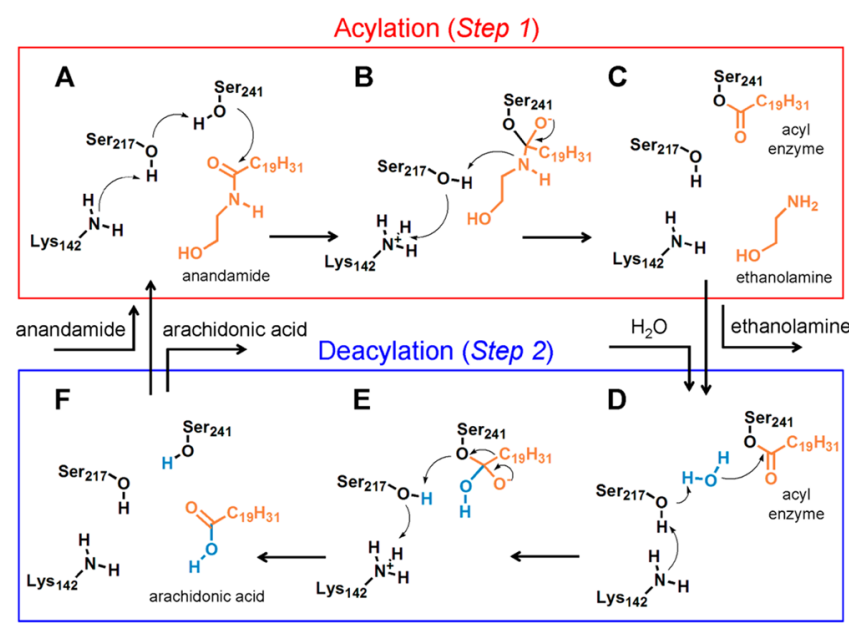

${ }^{a}$ The catalytic reaction has two main steps: formation of the acylenzyme complex (Acylation or Step 1 - top), and subsequent deacylation through a water molecule that enters into the catalytic cycle (Deacylation or Step 2 - bottom). Atoms belonging to the protein, anandamide, and the deacylating water molecule are represented in black, orange, and cyan, respectively. 
mechanism. ${ }^{17,18}$ Previously, the groups of Mulholland ${ }^{19-27}$ and Jorgensen $^{28-30}$ independently modeled FAAH acylation by a secondary substrate, i.e., oleamide-the primary amide of oleic acid-using different semiempirical quantum mechanics/ molecular mechanics (QM/MM) approaches. ${ }^{31}$ The acylation step was shown to occur in a concerted or quasi-concerted process, involving proton transfers that facilitate the nucleophilic attack of the activated Ser241 on the carbonyl carbon of the scissile amide bond to form the acyl-enzyme adduct (A$\mathrm{B}-\mathrm{C}$ in Scheme 1). Later, recent X-ray structures of FAAH, carbamoylated by different covalent inhibitors, were used by Mulholland et al. to investigate the FAAH deacylation reaction. ${ }^{16,22}$ These informative studies confirmed the overall mechanistic hypothesis, in which a water molecule acts as the nucleophilic agent attacking the acyl-enzyme adduct, with formation of the final product in which the catalytic triad is restored in its initial protonation state (D-E-F in Scheme 1).

It has been recently suggested ${ }^{32}$ that Nature evolved two catalytic strategies to facilitate enzyme-assisted amide bond hydrolysis through inversion of the scissile nitrogen (Scheme 2 ). Nitrogen inversion weakens the conjugation of the nitrogen

Scheme 2. (A) Crucial Conformational Changes of the Amide Bond That Reduces Conjugation of the Nitrogen Lone Pair with the Carbonyl, Activating the Substrate toward Nucleophilic Attack; (B) Proposed Catalytic Strategies for Enzyme-Assisted Amide Bond Hydrolysis via the Inversion of the Reactive Nitrogen ${ }^{a}$

A

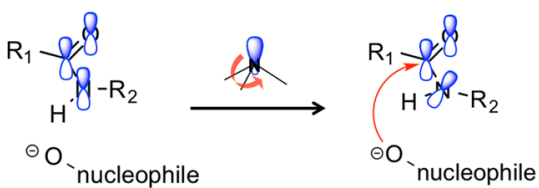

B

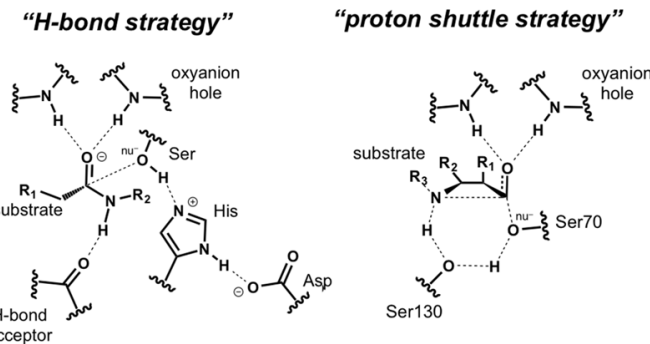

${ }^{a}$ In the "H-bond strategy" (left - shown for a generic Ser-His-Asp enzyme), an $\mathrm{H}$-bond acceptor, belonging either to the enzyme or to the substrate, facilitates the reactive nitrogen inversion by $\mathrm{H}$-bonding the NH amide group at the transition state (TS). In the "proton transfer strategy" (right - shown for class A of $\beta$-lactamases), the nucleophilic attack of Ser70 on the $\beta$-lactam carbonyl occurs concertedly with the protonation of the scissile $\beta$-lactam nitrogen by Ser130. Adapted from ref 32.

lone pair with the carbonyl group, thus overcoming the Deslongchamps stereoelectronic constraints that prevent the protonation and exit of the leaving group. ${ }^{33}$ In the first strategy-namely "H-bond strategy" - an H-bond acceptor that belongs either to the enzyme or to the substrate facilitates nitrogen inversion by $\mathrm{H}$-bonding the $\mathrm{NH}$ amide group in the rate-limiting transition state (TS). This mechanism is assisted by an oxyanion hole that is preorganized to stabilize the negatively charged substrate carbonyl at the TS. ${ }^{34}$ In this way, these simultaneous interactions that involve the amide functionality weaken the conjugation of the nitrogen lone pair with the carbonyl, leading to more efficient hydrolysis of the amide bond. ${ }^{10,35}$ Force-field-based methods and ab initio gas phase calculations on small model enzymatic systems have suggested that enzymes such as amidases and proteases, which share the above-mentioned structural architecture, facilitate the inversion of the scissile nitrogen for a facile breakdown of the amide functionality. ${ }^{32}$ Interestingly, these enzymes also share a conserved catalytic triad/dyad as engine of the catalytic mechanism. The second "proton shuttle strategy" - suggested for class $\mathrm{A}$ of $\beta$-lactamases that hydrolyze the cyclic amide moiety of penicillins-involves a concerted proton transfer mechanism within the catalytic residues that, during the nucleophile attack, simultaneously leads to the inversion and protonation of the scissile nitrogen. ${ }^{36,37}$ In the case of FAAH, the overall active site architecture suggested that the enzyme could perform amide bond hydrolysis via an "H-bond strategy" in which the carbonyl oxygen of Met191 H-bonds the scissile nitrogen, while the oxyanion hole chelates the substrates carbonyl. Although this structural evidence allowed the recognition of a plausible "H-bond strategy" for amide bond hydrolysis in FAAH, the exact mechanism and relevance of how the enzyme assists amide bond cleavage through the inversion of the scissile nitrogen has remained largely elusive. ${ }^{25,32}$

Through the integration of classical molecular dynamics (MD) and first-principles-based QM/MM MD simulations, we employ here a multiscale approach that depicts the full sequence of chemical and physical events of the enzymatic cycle. Based on our recent classical $\mathrm{MD}$ study on the mechanism of anandamide binding in $\mathrm{FAAH}^{14}{ }^{14}$ we investigate the complete catalytic cycle of FAAH in complex with its main substrate, providing a comprehensive description of both the acylation and deacylation reactions. In particular, we provide a detailed understanding of the nitrogen inversion mechanism for efficient amide bond hydrolysis, clarifying the mechanistic and energetic aspects of this crucial event. We also describe the exit of the leaving group through the "cytosolic port", providing the mechanistic connection the acylation and deacylation chemical steps.

The efficient enzyme-assisted amide bond hydrolysis in FAAH is characterized by a highly concerted multievent reaction sequence. We found that a critical contribution to catalytic efficiency enhancement originates indeed from the pyramidal inversion of the reactive nitrogen of the scissile amide bond in anandamide, which is a crucial structural change that drives the rate-determining acylation step to completion. This nitrogen inversion during amide bond hydrolysis in FAAH is aided by key structural elements present in the active site, which facilitate a cohesive sequence of chemical events. Overall, our results suggest that FAAH unifies the two canonical " $\mathrm{H}$ bond" and "proton-shuttle" strategies into a novel and dual approach for enzyme-assisted amide bond cleavage. This combined enzymatic strategy for amide bond hydrolysis might be general to a wide range of other amidases and proteases that share similar architectures of active site residues, as discussed herein.

\section{MATERIALS AND METHODS}

Structural Model. Calculations were based on the crystallographic structure of the rat FAAH protein, solved at $2.8 \AA$ resolution (PDB code: $1 \mathrm{MT5}),{ }^{12}$ in complex with the anandamide analogue methoxy arachidonyl fluorophosphonate (MAFP). The simulated system was composed of the FAAH/ 
anandamide complex embedded in an explicit membrane environment of 480 1-palmitoyl-2-oleoyl-phosphatidylethanolamine (POPE) lipids hydrated by means of $\sim 35500$ TIP3P ${ }^{38}$ waters. Eight $\mathrm{Cl}^{-}$counterions were added to neutralize the total charge. The size of the final system was $\sim 145 \AA \times \sim 95 \AA \times$ $\sim 140 \AA$, containing a total number of $\sim 185000$ atoms. Further details on the initial structural model are reported in our previous paper. $^{14}$

MD Simulations. Extensive $(\sim 1.5 \mu \mathrm{s})$ classical MD simulations on the FAAH/anandamide complex ${ }^{14}$ provided a suitable model of the Michaelis-Menten complex for subsequent Car-Parrinello (CP) QM/MM-MD simulations. $^{39}$ The all-atom AMBER/parm $99^{40}$ force field was adopted for the FAAH protein, whereas force field parameters for the lipid bilayer were identical to those used in our previous studies. ${ }^{14}$ The anandamide residue was treated with the General Amber Force Field (GAFF) ${ }^{41}$ and the atomic charges were derived by the RESP fitting procedure. ${ }^{42}$ Force field parameters for the non-standard residue were carefully validated via electronic structure calculations. ${ }^{14}$ The LINCS $^{43}$ algorithm was used to constrain covalent bonds including hydrogens, allowing an integration time step of 2 fs. The GROMACS $4^{44}$ code was used for the MD engine. The simulations were performed with deprotonated Lys142. The protonation state of Lys 142 has been proven via mutagenesis and kinetic experiments, further validated by a theoretical study, ${ }^{38}$ to be crucial for the catalytic mechanism of FAAH. ${ }^{12,15}$ Standard protonation states were maintained for the other protein residues. Approximately $\sim 1.5 \mu$ s of $\mathrm{MD}$ simulations were collected in the NPT ensemble under standard conditions. Details on the MD simulations can be found in the Supporting Information (SI) and in our previous paper. ${ }^{14}$

QM/MM-MD Simulations. The reaction paths were investigated via Car-Parrinello (CP) $\mathrm{QM} / \mathrm{MM}-\mathrm{MD}$ simulations ${ }^{45}$ that were carried out with the CPMD code. ${ }^{39,46}$ The reactive region of the complex, including anandamide and part of the catalytic triad residues Ser241, Ser217, and Lys142 (81 atoms in total) was treated at the DFT level of theory, using the BLYP functional, ${ }^{47,48}$ which has been shown to accurately describe a variety of enzymatic reactions. ${ }^{49-51}$ The remaining part of the system, including water molecules and counterions, as well as the POPE lipids, was treated at the classical level (AMBER force field). The terminal QM atoms (C $\beta @ S e r 241$, $\mathrm{C} \beta @$ Ser217, and $\mathrm{C} \delta(\mathrm{Lys} 142)$ were described with a monovalent pseudopotential. ${ }^{52}$ The wavefunctions were expanded in a plane wave basis set up to a cutoff of $75 \mathrm{Ry}$ in a QM cell of dimensions $18.0 \times 26.6 \times 22.0 \AA^{3}$ The interactions between the valence electrons and ionic cores were described with norm-conserving Martins-Troullier pseudopotentials. ${ }^{53}$ The QM part was treated as an isolated system, and electrostatic interactions between periodic images were decoupled by the scheme of Hockney. ${ }^{54}$ Notably, a rigorous Hamiltonian treatment of the electrostatic interaction between the QM and MM regions was used. ${ }^{45}$ This method has already been applied with success to a variety of enzymatic systems. ${ }^{55-57}$

The QM/MM protocol consisted of an initial optimization of the wavefunction, followed by $\sim 10$ ps of equilibration carried out with Born-Oppenheimer (BO) $\mathrm{MD}$ in the canonical (NVT) ensemble using an integration time step of $20 \mathrm{au}$ ( $\sim 0.48 \mathrm{fs})$. This method has been shown to be efficient for the $\mathrm{QM} / \mathrm{MM}$ equilibration phase of large biological systems ( 200 000 total atoms). ${ }^{58}$ The temperatures of the QM and
MM subsystems were kept constant at $300 \mathrm{~K}$ using a NoséHoover thermostat. ${ }^{59,60}$ After this initial phase, CP QM/MM simulations were carried out with a time step of 4 au $(\sim 0.097$ fs) and a fictitious electron mass of 400 au. The system was further equilibrated for $\sim 8$ ps.

One structure from the equilibrated CP QM/MM simulations was used for constrained $\mathrm{QM} / \mathrm{MM}$ simulations along a chosen reaction coordinate (RC). The free energy profile (FEP) for both acylation (Step 1, Scheme 1) and deacylation (Step 2) reactions of the catalytic cycle was obtained via thermodynamic integration (TI). So called "bluemoon" ensemble simulations were carried out adiabatically, constraining the $\mathrm{RC}$ at various distances, leaving all other degrees of freedom free to evolve. Several RCs were attempted for each chemical step, until a suitable one was identified (see more details in the Results and Discussion section, and the SI). The catalytic reaction pathways from reactants to products were divided in several TI windows with a resolution of $0.2 \AA$, respectively, and $0.1 / 0.05 \AA$ in the vicinity of the TS. In each TI step, simulations were performed for $\sim 2.5$ ps $(\sim 3.5$ ps at the top of the barriers). Data collection for the analysis were collected over the last $\sim 2 \mathrm{ps}$, in each window of the TI. The free energy profile was then obtained by integrating the average forces at every point along the RC. The error associated with each step of the TI was calculated by propagating the error on the forces at every step, using the propagation of error formula for linear functions. The error in the calculated free energy barrier was calculated as statistical error on the forces within each TI window. The obtained free energy profiles should be considered approximate due to the still limited sampling accessible to first-principles DFT calculations, the choice of a one-dimensional RC, and the limitations of the generalized gradient approximation (GGA) exchange-correlation functional (XC) BLYP. The catalytic pathways were then characterized in terms of variation of critical bond lengths and angles averaged over the equilibrated trajectory of each step of the constrained CP QM/MM simulations. Finally, the free energy cost related to the reactive nitrogen pyramidalization during enzyme acylation (Step 1 of the catalytic cycle) was explicitly evaluated using constrained dynamics (full details are reported in the SI). A total of $\sim 120$ ps of $\mathrm{QM} / \mathrm{MM}$ dynamics, including the equilibration time, were simulated.

\section{RESULTS AND DISCUSSION}

Reactant State for Anandamide Hydrolysis. Using microsecond MD simulations of FAAH embedded in a realistic membrane/water environment, we have recently described the molecular mechanism through which FAAH positions the anandamide substrate for hydrolysis. ${ }^{14}$ We have shown that anandamide likely assumes catalytically relevant conformations required for hydrolysis by moving its flexible arachidonoyl tail in between the "membrane access" and "acyl-chain binding" channels of the catalytic site (Figure 1). This process is regulated by two aromatic residues located at the boundary between the two channels (i.e., Phe432 and Trp531), which regulate the substrate movements within the active site via a "dynamic paddle" mechanism in agreement with previous hypotheses based on experimental results. ${ }^{61}$ Overall, our previous findings corroborated and further clarified crucial aspects of the proposed mechanism of binding, providing a solid model for the reactant state. ${ }^{1,12,13}$ Consequently, one representative catalytically relevant conformation of the system has been extracted from classical $\mathrm{MD}$ and subjected to $\mathrm{CP}$ 
QM/MM-MD simulations. This conformation constituted the Michaelis-Menten complex for the study of the reaction pathway for anandamide hydrolysis in FAAH. Full details are reported in the SI and in our previous paper. ${ }^{14}$

Acylation Reaction (Step 1). The FEP for the acylation step of the enzymatic hydrolysis $(\mathrm{A} \rightarrow \mathrm{C}$ in Scheme 1$)$ was computed using constrained dynamics, as explained in the Methods section. Constrained CP QM/MM simulations of 2.5/3.5 ps were performed for 12 points of the $\mathrm{RC}$ throughout the [2.71$1.37 \AA]$ spatial window needed to move from the reactant to the acyl-enzyme adduct. After several attempts (see SI), we found that the distance $d 1$ between the anandamide carbonyl carbon (C@anandamide) and the hydroxyl oxygen of Ser241 (O@Ser241) was a suitable RC, leading the reaction to completion (providing reasonable energetics). The structural and energetic features of the catalytic reactions are summarized in Figures 2 and 3.

The shape of the FEP (Figure 3, top graph) is characterized by two minima: the reactant state $(\mathbf{R})$ that constitutes the Michaelis-Menten complex and the acyl-enzyme adduct, which is an intermediate state of the entire catalytic cycle (INTa). A transition state (TS1) maximum separates $\mathbf{R}$ and INTa. In the $\mathbf{R}$ state $(\mathrm{RC}=2.71 \AA$ ), the system is stabilized by

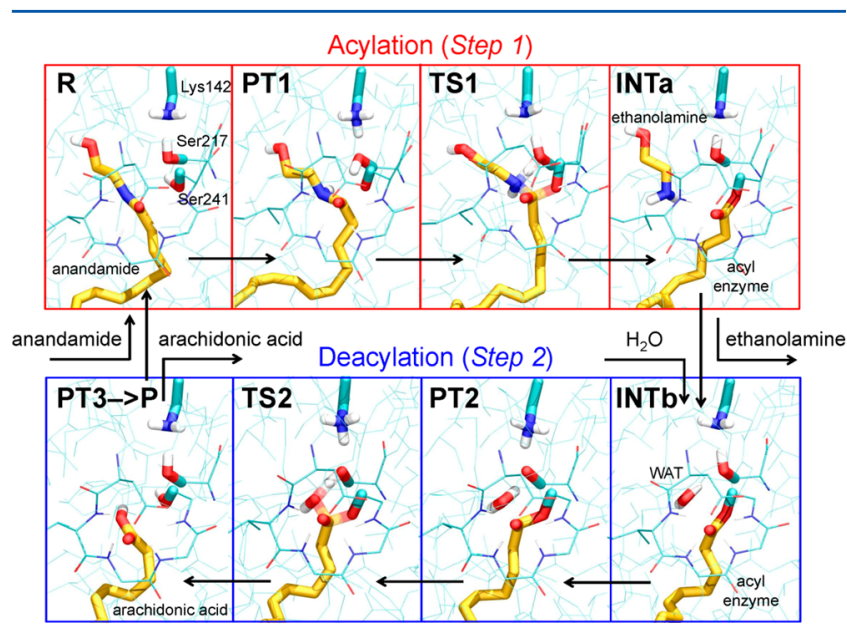

Figure 2. Selected snapshots from our $\mathrm{CP}-\mathrm{QM} / \mathrm{MM}$ simulations of the two investigated steps during the FAAH catalytic cycle for anandamide hydrolysis: acylation (Step 1 - top) and deacylation (Step 2 - bottom). Step 1 leads from the reactants (R) to the acylenzyme intermediate (INTa). A first proton transfer (PT1) within the Ser241-Ser217-Lys142 catalytic triad leads to the protonation of Lys 142 and to the simultaneous activation of Ser241 as a nucleophilic agent. The activated Ser241 attacks the substrate carbonyl (TS1). Here, a reverse proton transfer between Lys 142 and Ser217 allows the protonation of the leaving group ethanolamine, with formation of the acyl-enzyme intermediate (INTa). Step 2 starts from the hydrated acyl-enzyme adduct (INTb), obtained upon ethanolamine exit followed by the entrance of water molecules. A second proton transfer (PT2) allows the protonation of Lys142 by Ser217, which is deprotonated. At TS2, Ser217 can receive one proton from one deacylating water molecule (WAT), which is thus activated for the nucleophilic attack on the acyl-enzyme carbonyl. The reaction ends with a proton transfer (PT3) that reestablishes the original protonation state of the FAAH catalytic triad and leads to the formation of arachidonic acid as a final product of the catalytic mechanism (P). The enzyme is now ready for a new catalytic cycle. $\mathrm{QM}$ atoms of anandamide (yellow), the catalytic triad (cyan), and the deacylating WAT are represented with sticks. The surrounding residues - treated at MM level-are represented with thin cyan lines.

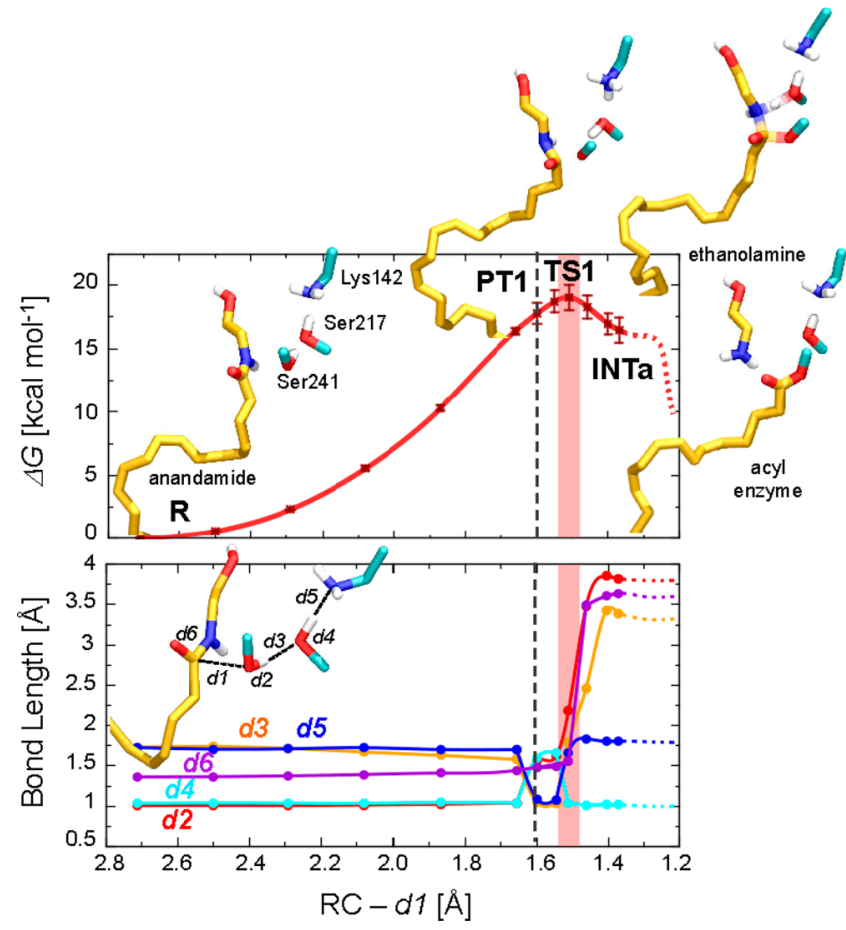

Figure 3. FEP (top) and selected average bond distances (bottom) along the acylation step (Step 1) of the catalytic cycle. The FEP is obtained via thermodynamic integration (TI), using the $d 1$ distance (C@anandamide-O@Ser241) as a RC. The transition state (TS1) region is highlighted in red. Selected snapshots along the RC are shown. At $\mathrm{RC}=2.71 \AA$, the system is in the reactant $(\mathrm{R})$ state. At RC $=1.60 \AA$, a concerted proton transfer (PT1) occurs within the catalytic triad, resulting in the protonation of Lys 142 and in the activation of Ser241 as a nucleophilic agent (i.e., an alkoxide species). At RC $=1.51$ $\AA$, TS1 is reached and a reverse proton transfer within the catalytic triad leads to the protonation of the anandamide nitrogen by Ser 217 . At RC $=1.37 \AA$, the system evolves toward the formation of the acylenzyme intermediate state (INTa) with the exit of an ethanolamine as a leaving group. QM atoms of anandamide (yellow) and the catalytic triad residues (cyan) are represented in sticks. Crucial $d 2-d 6$ bond distances (shown in the bottom graph) are defined as $d 2=\mathrm{O} @$ Ser241-H@Ser241, d3 = H@Ser241-O@Ser217, d4 = O@Ser217H@Ser217, d5 = H@Ser217-N@Lys142, d6=C@anandamide-N@ anandamide. Full details are reported in Figure S3.

a well-structured $\mathrm{H}$-bond network involving primarily the catalytic triad, in which Lys142 is deprotonated, as proposed for the reactants in FAAH catalysis. ${ }^{12,15}$ This H-bond network is well maintained until $\mathrm{RC}=1.66 \AA$.

At $\mathrm{RC}=1.60 \AA$, both $d 5$ (blue in Figure 3, bottom graph) and $d 3$ (orange) decrease, whereas $d 2$ (red) and $d 4$ (cyan) simultaneously increase, as a consequence of a proton transfer within the catalytic triad. In detail, Lys142 is protonated by Ser217 $(d 5=1.08 \pm 0.03 \AA)$ that, in turn, receives one proton from Ser241 $(d 3=1.04 \pm 0.03 \AA)$, which is approaching with its nucleophilic oxygen the anandamide carbonyl carbon to complete the nucleophilic attack with amide bond hydrolysis. This first concerted proton shuttle (PT1) does not lead to a stable intermediate, as observed in previous calculations for oleamide hydrolysis in FAAH. ${ }^{23,30}$ In fact, if the constraint on the $\mathrm{RC}$ is removed, the proton transfer is reversed and the system returns to $\mathbf{R}$.

At $\mathrm{RC}=1.51 \AA$, the first transition state (TS1) is reached, in which the average force on the $\mathrm{RC}$ constraint is zero. Here, Ser217 protonates the anandamide nitrogen after its inversion, 

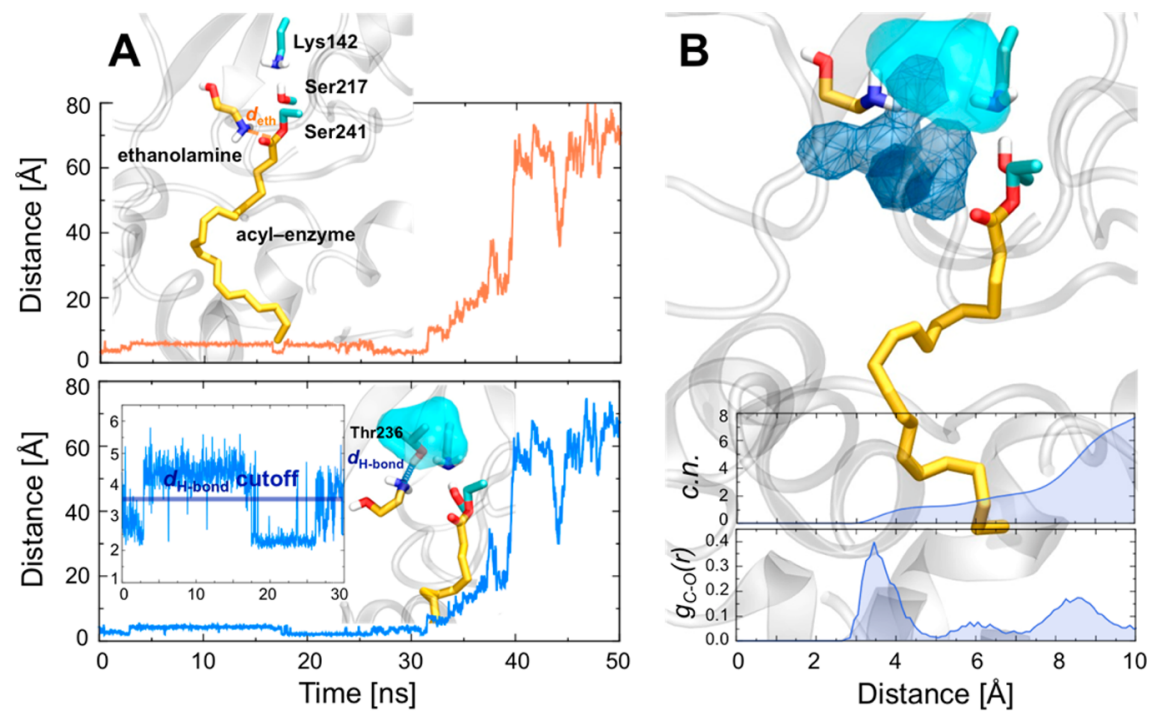

Figure 4. (A) Time evolution along $<50 \mathrm{~ns}$ of classical MD of the acyl-enzyme adduct (INTa) of the distance $d_{\text {eth }}$ between the ethanolamine nitrogen (N@ethanolamine) and the carbonyl carbon of anandamide covalently bound to Ser241 (C@anandamide-upper graph) and of the donor-acceptor H-bond distance $d_{\mathrm{H}-\text { bond }}$ between $\mathrm{N} @$ ethanolamine and $\mathrm{O} @ \mathrm{Thr} 236$ (lower graph). Time evolution of $d_{\mathrm{H} \text {-bond }}$ from 0 to $\sim 30 \mathrm{~ns}$ of $\mathrm{MD}$ is shown in the zoomed graph. A donor-acceptor H-bond distance cutoff equal to $\sim 3.5 \AA$ is highlighted as a blue bar. The initial MD configuration of INTa and the H-bond interaction between ethanolamine and Thr236 are shown at the top of the upper and lower graphs, respectively. Key protein residues (Ser241, Ser217, Lys142, and Thr236-cyan), the arachidonoyl chain and ethanolamine (yellow) are shown in sticks. The "cytosolic port" is represented with a cyan molecular surface. (B) Exit of the ethanolamine leaving group and hydration of FAAH catalytic site. Water density profiles (blue) generated as volumetric maps of the atomic coordinates of water molecules within $10 \AA$ of the anandamide carbonyl are shown, indicating the solvent accessible area (details are in the SI). The radial pair distribution function, $g_{\mathrm{C}-\mathrm{O}}(r)$, for water molecules within $10 \AA$ of the anandamide carbonyl carbon, calculated over $\sim 50 \mathrm{~ns}$ of MD trajectory of INTa, is reported in the bottom graph. The coordination number, c.n., is also reported in the top graph.

a prerequisite for proton abstraction (see next paragraph), which is therefore doubly protonated forming the ethanolamine leaving group. This proton transfer occurs simultaneously with a proton transfer from Lys142 to Ser217, which restores the initial protonation state of Lys142 and Ser217 (as $d 4=1.04 \pm$ $0.03 \AA$ and $d 5=1.67 \pm 0.09 \AA)$. These chemical events, i.e., proton transfers and amide bond breaking, are simultaneous in our simulations, leading to fast and complete amide bond hydrolysis and leaving group formation.

Acyl-Enzyme Adduct Hydrolysis. The acyl-enzyme adduct (INTa - Figure 2) contains the arachidonoyl chain of the anandamide substrate covalently bound to Ser241, while the leaving group ethanolamine is formed. This structure was equilibrated for $\sim 50 \mathrm{~ns}$ by means of classical $\mathrm{MD}$ simulations (details are reported in the Materials and Methods section and in the SI). After $\sim 27-30$ ns of classical dynamics, the ethanolamine leaving group spontaneously exits from the catalytic site through the cytosolic port (Figure 4), while the catalytic site is then filled by, on average, one water molecule. In detail, the distance $d_{\text {eta }}$ between the ethanolamine nitrogen ( $\mathrm{N} @$ ethanolamine) and the carbonyl carbon of anandamide now covalently bound to Ser241 (C@anandamide) increases up to $\sim 60-70 \AA$, resulting in the solvation of the ethanolamine residue. During the exit from the active site, the leaving group forms a H-bond with the cytosolic port residue Thr236 (Figure $4 \mathrm{~A}$, lower graph). As previously suggested, ${ }^{12,13,62}$ this interaction facilitates the leaving group departure from the FAAH active site. Concurrently, several water molecules fill the remaining enzymatic cavity, thus approaching the anandamide carbonyl for the acyl-enzyme deacylation. These results corroborate the mechanistic hypothesis of a crucial role of the cytosolic port in allowing both the exit of the leaving group from FAAH and, subsequently, the access of water molecules that can approach the acylated Ser241 for nucleophilic attack and acyl-enzyme adduct hydrolysis. ${ }^{12,13,16}$ Indeed, the radial pair correlation function $g_{\mathrm{C}-\mathrm{O}}(r)$ (Figure $4 \mathrm{~B}$ ) indicates that the probability of finding water molecules within $\sim 10 \AA$ to the anandamide carbonyl peaks around $3.20 \AA$, allowing the selection of one configuration for the subsequent CP QM/ MM simulations of the deacylation reaction.

Deacylation Reaction (Step 2). The deacylation reaction $(\mathrm{D} \rightarrow \mathrm{F}$ in Scheme 1) was simulated starting from the hydrated acyl-enzyme adduct INTb (Figure 2), obtained after classical MD simulations of INTa (i.e., the final acyl-enzyme adduct of the acylation reaction).

To characterize the deacylation step, we used the same procedure as for the previous acylation reaction. The distance $d 1^{\prime}$ between the carbonyl carbon of anandamide bound to FAAH (C@anandamide) and the oxygen of an additional water (O@WAT, now included in the QM part) turned out to be a suitable RC. We performed constrained CP QM/MM simulations for 13 points along the $\mathrm{RC}$, throughout the [3.10-1.40 $\AA]$ spatial window, leading to the final product.

The FEP of the deacylation reaction (Figure 5, top graph) is characterized by the hydrated acyl-enzyme adduct (INTb), the transition state TS2, and the final product $\mathbf{P}$. INTb $(\mathrm{RC}=3.10$ $\AA$ ) includes the nucleophilic WAT in a proper orientation for the nucleophilic attack on the anandamide carbonyl covalently bound to Ser241 (Figures 2 and 5). Moving from INTb to RC $=2.7$, we observed a stable H-bond between Ser217 and Lys142, while WAT remained correctly oriented for nucleophilic attack.

At $\mathrm{RC}=2.50 \AA$, Lys142 receives one proton from Ser217 (PT2). This is evidenced by $d 4^{\prime}$ (cyan), which increases to 1.41 $\pm 0.04 \AA$, whereas $d 5^{\prime}$ (blue) is reduced to $1.09 \pm 0.03 \AA$, at PT2. Releasing the constraints on the RC, PT2 falls back into 


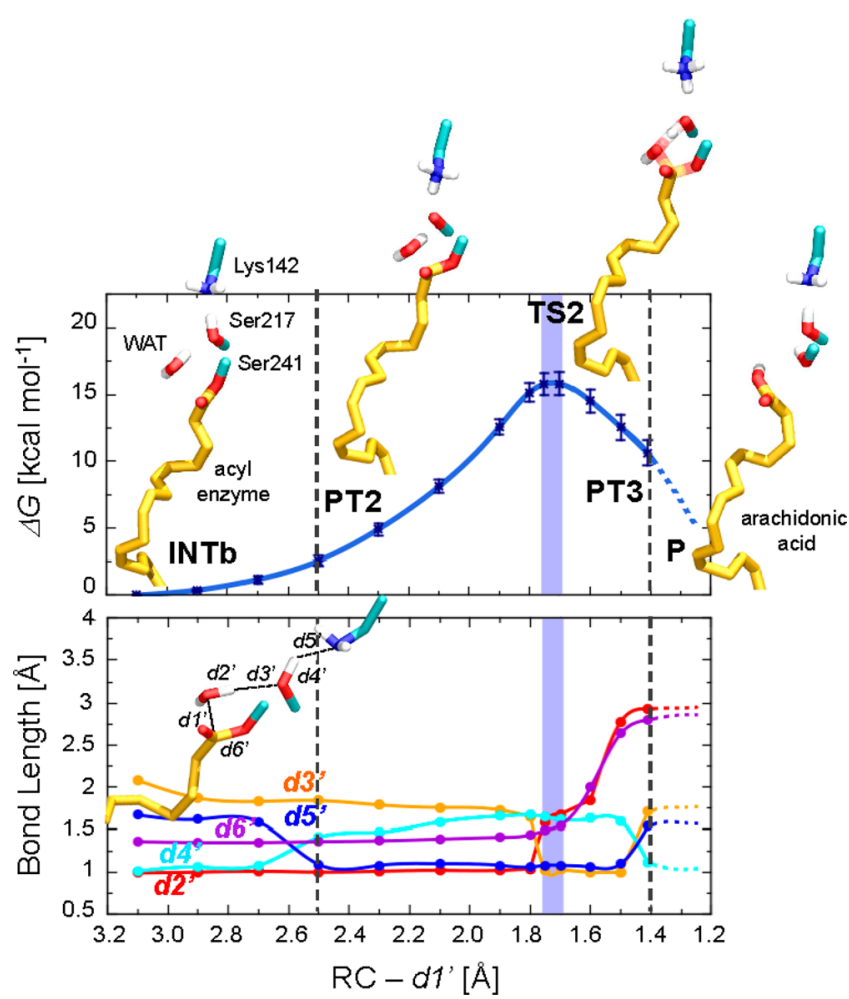

Figure 5. FEP (top) and selected average bond distances (bottom) along the deacylation step (Step 2) of the catalytic cycle. The FEP is obtained via thermodynamic integration (TI), using the $d 1^{\prime}$ distance (C@acyl-enyme-O@WAT) as a reaction coordinate (RC). The transition state (TS2) region is highlighted in blue. Selected snapshots along the $\mathrm{RC}$ are shown. At $\mathrm{RC}=3.10 \AA$, the acyl-enzyme intermediate (INTb) is well-positioned for its deacylation by a nucleophilic water (WAT). At RC $=2.50 \AA$, Lys 142 is protonated by Ser217 (PT1). At $1.75 \AA \geq R C \geq 1.70 \AA$, TS2 is reached. Here, WAT protonates Ser217, thus being activated as a nucleophilic species $\left(\mathrm{OH}^{-}\right)$for the attack on the acyl-enzyme carbonyl. At $\mathrm{RC}=1.40 \AA$, a proton transfer (PT3) leads to the restoration of the initial protonation state of the catalytic triad while the system evolves toward the product (P) state with the formation of arachidonic acid. QM atoms of arachidonoyl chain (yellow), of the catalytic triad residues (cyan), as well as of the deacylating WAT are represented in sticks. Crucial $d 2^{\prime}-d 6^{\prime}$ bond distances (show in the bottom graph) are defined as $d 2^{\prime}=\mathrm{O} @ W A T-H @ W A T, d 3^{\prime}=\mathrm{H} @ W A T-O @ S e r 217$, $d 4^{\prime}=\mathrm{O} @ S e r 217-\mathrm{H} @ S e r 217, d 5^{\prime}=\mathrm{H} @ S e r 217-\mathrm{N} @ L y s 142, d 6^{\prime}=$ C@acyl-enzyme-O@Ser241. Full details are reported in Figure S4 (SI).

the INTb minimum, suggesting that PT2 is not a stable intermediate along the catalytic path.

At $\mathrm{RC} \in[1.70,1.75] \AA$, the average force on the $\mathrm{RC}$ constraint is zero, indicating that a transition state (TS2) for the deacylation reaction is reached. Within this narrow interval, $d 2^{\prime}$ (red) increases to $1.68 \pm 0.06 \AA$, whereas $d 3^{\prime}$ (orange) decreases to $1.03 \pm 0.04 \AA$, as a consequence of the proton transfer between WAT and the alkoxide group of Ser217. The deprotonated WAT (now $\mathrm{OH}^{-}$) can therefore readily attack the anandamide carbonyl carbon covalently bound to Ser241, leading to hydrolysis of the acyl-enzyme adduct and formation of the final leaving group, i.e., the arachidonic acid.

At $\mathrm{RC}=1.60 \AA$, the average constraint force changes sign, indicating that the system has crossed TS2, downhill toward the product state $(\mathbf{P})$. Here, $d 6^{\prime}$ (violet) progressively increases, as the leaving arachidonic acid is getting away from the catalytic
Ser217. At RC $=1.40 \AA$, a final proton shuttle (PT3) restores the starting protonation state of the catalytic triad. In detail, Lys 142 transfers one proton to Ser217 $\left(d 5^{\prime}=1.54 \pm 0.05 \AA\right.$; $d 4^{\prime}=1.11 \pm 0.06 \AA$ ) which, in turn, protonates Ser241. At this point, upon removal of the constraints, the system reaches a final state $\mathbf{P}$ where $\mathrm{RC}$ is $\sim 1.37 \AA$.

As for the acylation step, the series of chemical events that include proton transfers and acyl-enzyme hydrolysis (in PT2TS2-PT3) is highly concerted, although asynchronous, with no detectable (meta)stable intermediates.

Energetics along the Catalytic Cycle. The FEPs of the two steps of the enzymatic reaction-i.e., acylation (Step 1) and deacylation (Step 2)-were calculated by integrating the average force of constraint as described in the Materials and Methods section and plotted in Figures 3 and 5, respectively. The calculated free energy barrier for the acylation reaction is $19.0 \pm 1.2 \mathrm{kcal} \mathrm{mol}^{-1}$, while the calculated free energy barrier for the deacylation reaction is $16.0 \pm 1.0 \mathrm{kcal} \mathrm{mol}^{-1}$. Our findings are consistent with the experimental value related to the overall catalytic rate $\left(k_{\text {cat }}=14.4 \pm 0.63 \mathrm{~s}^{-1}\right)$ that, employing transition state theory assuming a transmission factor of unity, results in an activation barrier of the rate limiting step of $\sim 16$ $\mathrm{kcal} \mathrm{mol}^{-1} \cdot{ }^{63-65}$ If we assume that the two steps of the catalytic cycle are sufficiently disconnected by the relatively slow replacement of the leaving group (ethanolamine in INTa) with water molecules hydrating the remaining catalytic cavity, INTb can be considered a new equilibrated reference state from which the system can evolve to reach the final $\mathbf{P}$ state. ${ }^{57}$ Therefore, given the lower calculated free energy barrier for the deacylation reaction $\left(\sim 16 \mathrm{kcal} \mathrm{mol}^{-1}\right)$, with respect to the acylation reaction $\left(\sim 19 \mathrm{kcal} \mathrm{mol} \mathrm{m}^{-1}\right)$, the latter can be considered to be the rate-determining step of the entire chemical process, in agreement with previous studies. $^{19,21-23,25-27,30}$

Overall, our results indicate a single-step mechanism for both acylation and deacylation reactions in FAAH. This is a highly concerted mechanism, where spontaneous and complete proton transfers without stepwise intermediate states facilitate the nucleophilic attacks and leaving group departures. During both steps, we observe proton transfers (PT1 and PT2) that precede the formation of transition states TS1 and TS2, while PT3 follows TS2, leading to the final state P. Therefore, PT1, PT2, and PT3 are concerted asynchronous events needed for nucleophilic attack in TS1 and TS2. Also, these proton shuttles, which are necessary for nucleophile formation and leaving group departure, are observed spontaneously, suggesting small energetic barriers that could be further reduced by quantum effects, such as tunneling, which are neglected in our calculations. Although we cannot rule out a possible stepwise mechanism for the observed sequence of proton transfers, our results suggest that the two chemical steps happen without formation of metastable intermediates. This indicates a highly concerted multi-event mechanism for both acylation and deacylation steps.

Our computations agree well with previous computational results on FAAH reactivity with secondary oleoyl substrates. Indeed, the acylation step of oleamide hydrolysis (Step 1) was first modeled by Lodola et al., using QM/MM minimizations in an adiabatic mapping approach. ${ }^{23}$ These semiempirical calculations, corrected via DFT single point calculations, indicated that the proton exchange within the catalytic triad is simultaneous to the formation of a tetrahedral intermediate $(\mathrm{A} \rightarrow \mathrm{B})$, with a calculated energy barrier of $\sim 19 \mathrm{kcal} \mathrm{mol}^{-1}$ 
(B3LYP $66-68 / 6-31+\mathrm{G}(\mathrm{d}) / /$ PM3-CHARMM). This process has been shown to be slightly favored $\left(\sim 2 \mathrm{kcal} \mathrm{mol}^{-1}\right)$ with respect to the subsequent formation of the acyl-enzyme (B$\mathrm{C})$, suggesting a concerted acylation step of oleamide hydrolysis in FAAH. ${ }^{19,23,25}$ These results agree also with the study of Tubert-Brohman et al..$^{30}$ who, through Monte Carlo $\mathrm{QM} / \mathrm{MM}$ calculations at the semiempirical PDDG/PM3 level, confirmed a concerted mechanism for the acylation process for the oleoyl substrates. Lodola et al. also investigated the deacylation of FAAH, acylated by oleamide and few carbamate and urea-based covalent inhibitors. ${ }^{22}$ These QM/MM calculations (SCC-DFTB/CHARM27) have shown that a mechanism involving a water molecule as a nucleophile leads to the formation of a tetrahedral intermediate structure (E, Scheme 1 ), with an overall barrier of $\sim 16 \mathrm{kcal} \mathrm{mol}^{-1}$. Interestingly, the fact that less concerted hydrolytic mechanisms were found for less specific FAAH substrates ${ }^{3,69}$ supports the high catalytic efficiency of FAAH in hydrolyzing its main substrate anandamide through a highly concerted mechanism, as described in this study.

Nitrogen Inversion in Anandamide. We found that a crucial event for anandamide hydrolysis is the pyramidal inversion of the reactive nitrogen of the scissile amide bond. This event occurs during the rate-limiting acylation reaction (Step 1) and is key in favoring the formation of the leaving group, as it properly orients the nitrogen lone pair toward Ser217 for the protonation and subsequent exit of the leaving group (Figure 6, upper panel).

The nitrogen pyramidalization and inversion along the reaction is evidenced by the probability density functions of the improper torsion angle $\theta$ centered on the anandamide nitrogen (Figure 6; probability distributions of $\theta$ along the RC represented as a function of colors red $\rightarrow$ blue $\rightarrow$ black). Up to $\mathrm{RC}=1.55 \AA$, $\theta$ preferentially assumes negative values (red $\rightarrow$ blue distributions), which correspond to conformations of anandamide prone to undergo the nucleophilic attack by Ser241 (PT1 in Figure 6). In fact, the nitrogen lone pair points in the opposite direction with respect to the nucleophilic Ser241, and allows the formation of a tetrahedral carbonyl. However, these conformations do not permit the necessary protonation and exit of the ethanolamine leaving group. To overcome this issue, at TS1, a fast pyramidal inversion of the reactive nitrogen properly orients its lone pair toward Ser217 (TS1 in Figures 3 and 6). When TS1 is reached, in fact, we observed a rapid shift of $\theta$, from negative to positive values $(\theta$ $\sim 20^{\circ}$, gray), which indicates the nitrogen inversion, preluding to the proton abstraction by the reacting nitrogen for leaving group formation and proceeding of catalysis. Electronic density $(\rho)$ distributions on the reactive nitrogen of anandamidecalculated for selected snapshots along the reaction (Figure 6) - are further evidence of the nitrogen inversion at TS1, which now points in the direction of Ser217. This is reflected by the $\theta$ angle, which is now stabilized around $30^{\circ}$ (purple distributions when $\mathrm{RC} \in[1.40,1.46] \AA)$. At $\mathrm{INTa}(\mathrm{RC}=1.37$ $\AA)$, the ethanolamine leaving group is free to move away from the catalytic triad, resulting in a broad probability distribution of the $\theta$ angle (black distribution curve).

We estimated the free energy barrier for the anandamide nitrogen inversion during FAAH acylation to be $\sim 4.5 \pm 0.7$ $\mathrm{kcal} \mathrm{mol}^{-1}$ (see Figure 7A and SI for details). Typical values for the nitrogen inversion are between 4 and $15 \mathrm{kcal} \mathrm{mol}^{-1}$, ${ }^{32}$ suggesting a rather low cost for the nitrogen inversion during anandamide hydrolysis. The energetics for this necessary event,
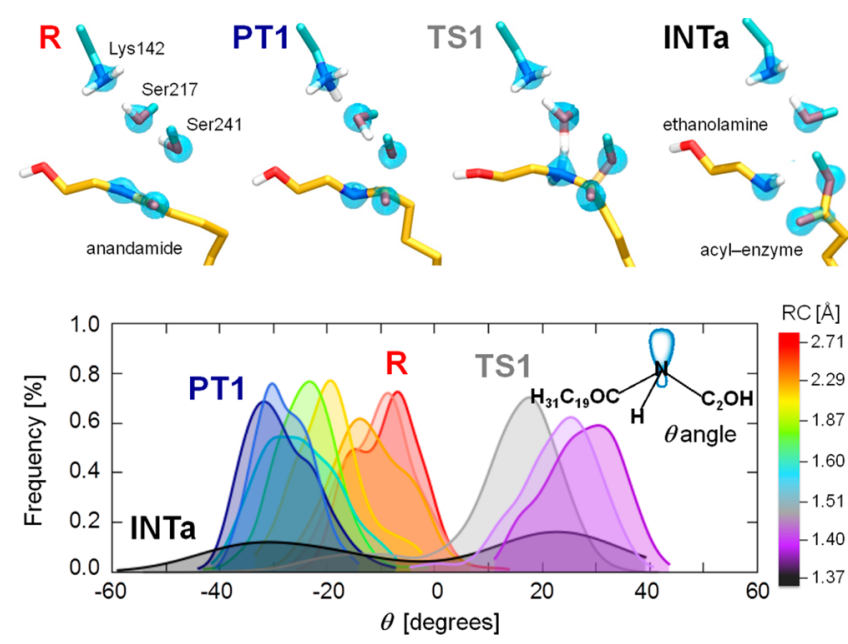

Figure 6. Probability distribution functions of the improper torsion angle centered on the anandamide nitrogen $(\theta-$ on the right top of the graph) are shown for different points along the RC, during Step 1 of the catalytic cycle. Distribution curves are shown using a color scale, ranging from the reactants $(\mathrm{R}-$ red), to the proton transfer 1 (PT1 blue), the transition state 1 (TS1 - gray) and to the acyl-enzyme intermediate (INTa - black) states. The height of the distribution curves represents the probability (i.e., frequency, expressed in \%) that the $\theta$ angle assumes a certain value, whereas the spread of the distribution curves denotes the magnitude in fluctuation of the $\theta$ angle. Kernel density estimation functions have been used to estimate the probability distribution of the $\theta$ angle at each point along the RC (details are reported in the SI). At TS1, a shift of the probability distribution of $\theta$ from negative to positive values indicates a rapid inversion of the reactive nitrogen configuration, which is needed for the protonation and exit of the leaving group. Electronic density $(\rho)$ distributions (at the very top), calculated for crucial points along the RC, show a shift of the $\rho$ centered on the reacting nitrogen at the TS1. As a consequence of the pyramidal inversion of the nitrogen at TS1, the $\rho$ centered on the reacting nitrogen points toward Ser217, indicating the favorable orientation of the nitrogen lone pair for the protonation and the subsequent exit of the ethanolamine leaving group. QM atoms of anandamide (yellow) and the catalytic triad residues (cyan) are represented in sticks. $\rho$ distributions are shown as a cyan surface (shown with an isovalue of 0.2 electrons $/ \AA^{3}$ ).

however, should be considered as the last kick in energy needed to overcome TS1 for complete amide bond cleavage and leaving group formation. ${ }^{32,70}$ In addition, we estimated the barrier for nitrogen inversion during anandamide hydrolysis in water (see SI for details), using a nucleophilic water for the attack on the carbonyl carbon of anandamide. As expected, the barrier for nitrogen inversion is much higher $\left(\sim 16 \mathrm{kcal} \mathrm{mol}^{-1}\right)$ in water than in the enzyme, which further demonstrates the role of the enzymatic structure in favoring nitrogen inversion and protonation. Indeed, when the nucleophilic oxygen attacks the amide bond, the $\mathrm{sp} 2$ character of the carbonyl group forming the amide bond is lost in favor of a tetrahedral carbon, typical of sp3 hybridization. In this conformation (see snapshot PT1 along FAAH catalysis in Figures 6 and 7), the inversion of the reactive nitrogen is difficult, although necessary for leaving group protonation and exit.

It has been recently suggested that several amidases and proteases have evolved two basic strategies to efficiently catalyze amide bond hydrolysis via the inversion of the scissile nitrogen configuration: (i) in the "H-bond strategy" an H-bond acceptor facilitates nitrogen inversion by $\mathrm{H}$-bonding the $\mathrm{NH}$ amide group of the scissile amide, while a oxyanion hole 
A

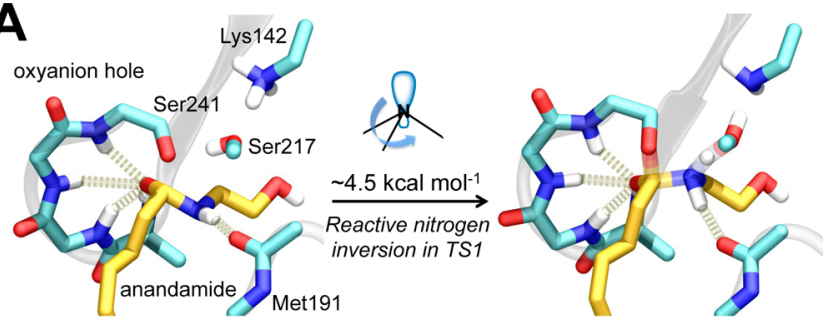

B

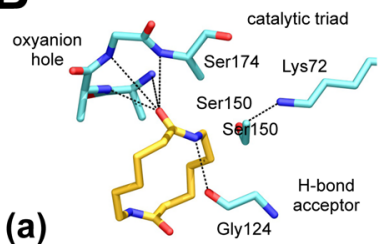

(a)
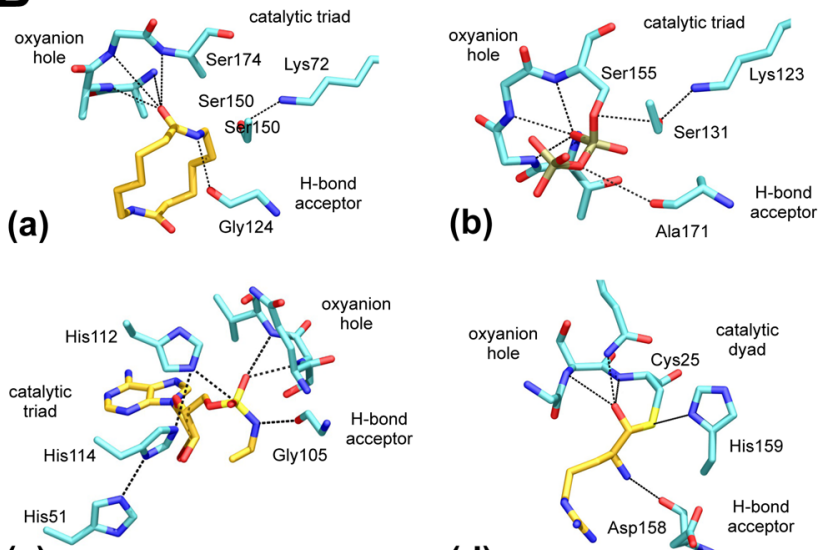

(c)

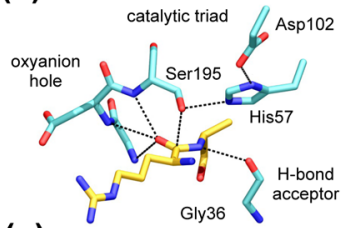

(e)

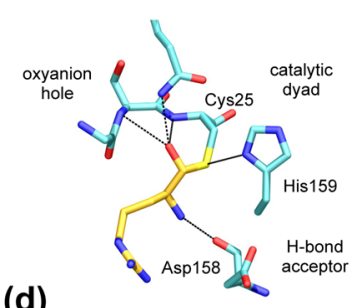

(d)

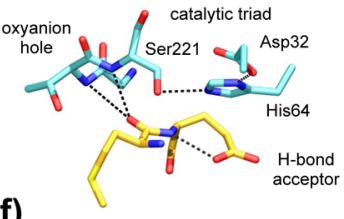

(f)

Figure 7. (A) Mechanism for the scissile nitrogen inversion in FAAH from $\mathrm{QM} / \mathrm{MM}$ simulations. Pyramidalization (left) and inversion (right) of the reactive nitrogen of anandamide, occurring during the proton transfer (PT1) and the TS1 of the rate-limiting acylation reaction in FAAH catalysis, respectively. Anandamide is shown in yellow sticks. The catalytic triad (Ser241-Ser217-Lys142), the oxyanion hole (Ile238, Gly239-Gly240-Ser241), and Met191 are shown in cyan sticks. Crucial H-bonds, formed between the $\mathrm{NH}$ group of the scissile amide bond and the backbone oxygen of Met191, as well as between the anandamide carbonyl oxygen and the oxyanion hole residues, are highlighted. The energetic cost for the pyramidal inversion of the reactive nitrogen was calculated to be $\sim 4.5 \mathrm{kcal}$ $\mathrm{mol}^{-1}$. (B) Catalytic site of some relevant amidases and proteases: (a) 6-aminohexanoate cyclic dimer hydrolase, ${ }^{87}$ (b) malonamidase, ${ }^{73}$ (c) Hint AMP-lysine hydrolase, ${ }^{97}$ (d) papain, ${ }^{79}$ (e) trypsin, ${ }^{75}$ and (f) subtilisin. ${ }^{98}$ These enzymes share the crucial structural features used in FAAH to facilitate amide bond cleavage. This consensus, a catalytic triad/dyad as the engine of the catalysis, an "oxyanion hole" type network properly oriented for the stabilization of the transition state, an H-bond acceptor, which belongs either to the enzyme $(\mathrm{a}-\mathrm{e})$ or to the substrate (f). Protein residues (cyan) and substrates (yellow) and an H-bond network around the scissile nitrogen are shown in sticks. A full comparison with the enzymes of the classes of amidases and proteases is reported in the SI.

stabilizes the negatively charged substrate carbonyl at the TS; (ii) in the "proton shuttle strategy" a concerted proton transfer within the catalytic residues simultaneously allows for the inversion and protonation of the scissile nitrogen (Scheme 2). ${ }^{32}$ Based on the structural characteristics of FAAH active site, which is composed by a conserved oxyanion hole and by a Met191 carbonyl oxygen in close contact with the NH of the scissile amide, it has been suggested that FAAH employs an " $\mathrm{H}$ - bond strategy" to facilitate the inversion of the scissile nitrogen and allow a facile breakdown of the amide functionality. ${ }^{32}$ Interestingly, the hypothesis of an enzyme-induced amide bond distortion to catalyze hydrolysis has been recently proposed to rationalize the mechanism used by cyclic piperidine/piperazinebased compounds to covalently inhibit FAAH. ${ }^{10,71}$ Classical $\mathrm{MD}$ simulations, combined with $\mathrm{QM} / \mathrm{MM}$ calculations, indicated that distorted amides are more susceptible to nucleophilic attack by Ser241, than their planar analogues [given a favored HOMO-LUMO energy gap in the reactant state of $\sim 0.35-0.40 \mathrm{eV}$ (i.e., $\sim 8-9 \mathrm{kcal} \mathrm{mol}^{-1}$ )]. ${ }^{10}$ However, these studies could not look at structural rearrangements during bond forming and breaking events, leaving mechanistic questions on how enzymes could promote catalysis via the induction of the reactive nitrogen distortion/inversion.

Our simulations show that the inversion of the reactive nitrogen in FAAH is facilitated by the surrounding residues, which establish an ordered H-bond network at TS1 (Figure $7 \mathrm{~A})$. Indeed, during the evolution of the acylation reaction from $\mathbf{R}$ to TS1, distances and angles for the oxyanion hole residues chelating the anandamide carbonyl assume favorable values for the proper stabilization of the negatively charged substrate carbonyl at TS1 (Figure S5-6). Concurrently, the NH group of the scissile amide bond coordinates with the backbone carbonyl oxygen of Met191. Hence, as recently suggested, ${ }^{32}$ FAAH seems to use an "H-bond strategy" to overcome the stereoelectronic constraints for nitrogen inversion. At the same time, our simulations show that the catalytic triad performs a concerted proton shuttle that, at TS1, transfers one proton from Ser 217 to the amide nitrogen, leading to the completeness of the reaction via a "proton shuttle strategy". Therefore, our $\mathrm{QM} / \mathrm{MM}$ simulations indicate that, beside the suggested " $\mathrm{H}$ bond strategy", FAAH simultaneously exploits a "proton shuttle strategy" for the accomplishment of a facile hydrolysis of the amide functionality. The overall strategy is therefore a combination of ordered H-bonds networks (i.e., "H-bond strategy") and simultaneous "proton shuttle" into a dual strategy which magnifies the catalytic efficiency of FAAH.

The inspection of the available crystallographic data (Figure $7 \mathrm{~B}$ and $\mathrm{SI}$ ) reveals that a wide range of amidases and proteases share the crucial structural features used in FAAH to facilitate amide bond cleavage. In fact, several other enzymes belonging to the amidase signature family, such as peptide amidase, ${ }^{72}$ malonamidase ${ }^{73}$ and others, ${ }^{32}$ show a conserved sequence of Ser-Ser-Lys catalytic residues flanked by an oxyanion hole and by a backbone carbonyl oxygen placed to form a H-bond with the scissile amide group. Beside the amidase signature family, other enzymes such as enteropeptidase ${ }^{74}$ and its activated product-the enzyme trypsin ${ }^{75}$ that is responsible for the metabolic control of digestion-as well as different oligopeptidases ${ }^{76,77}$ show this characteristic architecture with a Ser-His-Asp catalytic triad. Other relevant enzymes that are characterized by a similar active site framework to catalyze amide nitrogen inversion, and perform the amide bond hydrolysis via a catalytic dyad, include pepsin, ${ }^{78}$ one of the main enzymes involved in protein degradation, that acts via an Asp/Asp catalytic dyad and papain, ${ }^{19}$ an important cysteine protease of plants that employs a Cys/His catalytic dyad. A nucleophilic cysteine and an oxyanion hole architecture with Asp21 as an H-bond acceptor has also been described for the conjugated bile acid hydrolase catalytic machinery. ${ }^{80}$

This comparison with X-ray and NMR data allows the extension of the mechanistic hypothesis to other enzymes, such 
as class $\mathrm{A}$ of $\beta$-lactamases, ${ }^{36,37}$ carboxypeptidase $\mathrm{Y},{ }^{81}$ the aspartic proteinase ${ }^{82}$ and the matrix metalloproteinase MMP$8^{83}$ that has been solved in complex with an $N$-hydroxyurea inhibitor, showing a high out-of-plane distortion of the amide bond. It is interesting to note that many esterases and lipases acting on substrates that do not require nitrogen inversion for hydrolysis (i.e., esters and glycols), such as human liver carboxylesterase $1,{ }^{84}$ lipase B from candida antarctica ${ }^{85}$ and others, $^{32}$ are poor catalysts for amide bond hydrolysis. ${ }^{86}$ Although these enzymes share a functional catalytic triad and an oxyanion hole network with amidases and proteases, they lack an H-bond acceptor, which, in contrast, is conserved in many amidases and proteases. ${ }^{72-79,87}$

Taken together, these structural data indicate how a wide range of amidases and proteases could assume a catalytically competent state for the amide bond cleavage that agrees well with the mechanistic details discovered QM/MM simulations for anandamide hydrolysis in FAAH. Therefore, the catalytic mechanism employed by FAAH for the amide bond cleavage, which is described here as a dual " $\mathrm{H}$-bond/proton shuttle strategy" facilitating the scissile nitrogen inversion, could represent a general mechanistic approach for efficient enzyme-assisted amide bond hydrolysis in amidases and proteases.

Overall, while also reinforcing the hypothesis that an enzyme-induced amide bond distortion could favor the formation of covalent adducts in $\mathrm{FAAH},{ }^{10,71}$ our CP QM/ MM simulations now offer an atomistic description on how the crucial nitrogen inversion is facilitated during the acylation step of anandamide hydrolysis, providing new insights into the general mechanism of amide bond hydrolysis.

\section{CONCLUSIONS}

Here, we reported a comprehensive study of the entire cycle of FAAH catalysis, in which its main substrate anandamide is hydrolyzed through two steps, i.e., enzyme acylation and deacylation. Our first principles-based simulations have helped to decipher a highly concerted mechanism that explains the catalytic efficiency of this complex multievent reaction. Briefly, each step is characterized by proton shuttle events that happen concertedly with the nucleophilic attack, with no detectable intermediate along both steps. As a result, the acylation reaction is confirmed to be the rat-determining step of the overall hydrolytic process, in agreement with kinetic experiments and previous computational studies.

In addition, the combination of classical and first principlesbased QM/MM MD simulations has clarified the complete sequence of the chemical and physical events during enzymatic hydrolysis of anandamide into arachidonic acid and ethanolamine. Notably, we have quantitatively described a crucial event during the acylation rate-limiting step, which is the inversion of the reactive nitrogen of the scissile amide bond in anandamide. The stereoelectronic constraint for amide bond hydrolysis is overcome using an exquisite mechanism, which unifies the two canonical "H-bond" and "proton-shuttle" strategies into a novel and dual approach for enzyme-assisted amide bond cleavage.

Importantly, the same catalytic machinery used to induce amide bond distortion, nitrogen inversion and final amide bond cleavage seems to be exploited by several other amidases and proteases that present similar structural features of the catalytic site, ${ }^{32,72,88-93}$ thus suggesting a possible generalization of this mechanism for enzyme-assisted amide bond hydrolysis in the biology of amidases and proteases.
On the basis of these considerations, the on-the-fly description provided herein of how nitrogen inversion helps efficient hydrolysis of anandamide in FAAH extends the current knowledge on the enzymatic strategy for efficient enzymeassisted amide bond hydrolysis. Our first principles-based information could therefore encourage further experimental investigations to test the relevance of nitrogen inversion and validate our strategy for efficient enzyme-assisted amide bond hydrolysis in the amidases and proteases biology. Finally, these mechanistic insights might also help in de novo enzyme design $^{94}$ or drug discovery programs, ${ }^{95,96}$ inspiring the introduction of catalytic elements either in the enzyme structure or in the ligand scaffold in order to induce amide bond distortion and improve their catalytic/inhibitory efficiency.

\section{ASSOCIATED CONTENT}

\section{S Supporting Information}

Details on classical and QM/MM Car-Parrinello MD simulations. Description of the criteria for the selection of one of the representative catalytically relevant conformations, as initial model of the Michaelis-Menten complex for QM/MM simulations. Details on the parameters employed for all structural analyses of the classical and QM/MM trajectories. Full structural analyses are also reported. Energetics for anandamide nitrogen pyramidalization in FAAH and in solution. Detailed comparison of FAAH catalytic features with existing structural information on several amidases and proteases. RESP charges and parameters employed in classical $\mathrm{MD}$ for the nonstandard residues. Coordinates of the QM atoms for the transition state structures (i.e., TS1 and TS2 for the acylation and deacylation reactions, respectively). Two supplementary movies are provided: Movie 1 shows the entire reactive process (i.e., acylation and deacylation reactions), while Movie 2 clarifies how the two proposed "H-bond" and "protonshuttle" strategies are unified into a dual mechanism for amide bond cleavage and nitrogen inversion in FAAH. This material is available free of charge via the Internet at http://pubs.acs.org.

\section{AUTHOR INFORMATION}

\section{Corresponding Authors}

*E-mail: marco.devivo@iit.it.

*E-mail: ursula.roethlisberger@epfl.ch

\section{Notes}

The authors declare no competing financial interest.

\section{ACKNOWLEDGMENTS}

M.D.V. thanks the Italian Association for Cancer Research (AIRC) for the financial support through the Grant MFAG No. 14140. We thank A. Lodola, S. Meloni, and E. Liberatore for useful discussions.

\section{REFERENCES}

(1) Ahn, K.; McKinney, M. K.; Cravatt, B. F. Enzymatic Pathways that Regulate Endocannabinoid Signaling in the Nervous System. Chem. Rev. 2008, 108, 1687-1707.

(2) Piomelli, D. The Molecular Logic of Endocannabinoid Signalling. Nat. Rev. Neurosci. 2003, 4, 873-884.

(3) Desarnaud, F.; Cadas, H.; Piomelli, D. Anandamide Amidohydrolase Activity in Rat Brain Microsomes. Identification and Partial Characterization. J. Biol. Chem. 1995, 270, 6030-6035.

(4) Devane, W. A.; Hanus, L.; Breuer, A.; Pertwee, R. G.; Stevenson, L. A.; Griffin, G.; Gibson, D.; Mandelbaum, A.; Etinger, A.; 
Mechoulam, R. Isolation and Structure of a Brain Constituent that Binds to the Cannabinoid Receptor. Science 1992, 258, 1946-1949.

(5) Cravatt, B. F.; Giang, D. K.; Mayfield, S. P.; Boger, D. L.; Lerner, R. A.; Gilula, N. B. Molecular Characterization of an Enzyme that Degrades Neuromodulatory Fatty-Acid Amides. Nature 1996, 384, 83-87.

(6) Giang, D. K.; Cravatt, B. F. Molecular Characterization of Human and Mouse Fatty Acid Amide Hydrolases. Proc. Natl. Acad. Sci. U. S. A. 1997, 94, 2238-2242.

(7) Bertolacci, L.; Romeo, E.; Veronesi, M.; Magotti, P.; Albani, C.; Dionisi, M.; Lambruschini, C.; Scarpelli, R.; Cavalli, A.; De Vivo, M.; Piomelli, D.; Garau, G. A Binding Site for Nonsteroidal Antiinflammatory Drugs in Fatty Acid Amide Hydrolase. J. Am. Chem. Soc. 2013, 135, 22-25.

(8) Favia, A. D.; Habrant, D.; Scarpelli, R.; Migliore, M.; Albani, C.; Bertozzi, S. M.; Dionisi, M.; Tarozzo, G.; Piomelli, D.; Cavalli, A.; De Vivo, M. Identification and Characterization of Carprofen as a Multitarget Fatty Acid Amide Hydrolase/Cyclooxygenase Inhibitor. J. Med. Chem. 2012, 55, 8807-8826.

(9) Otrubova, K.; Brown, M.; McCormick, M. S.; Han, G. W.; O’Neal, S. T.; Cravatt, B. F.; Stevens, R. C.; Lichtman, A. H.; Boger, D. L. Rational Design of Fatty Acid Amide Hydrolase Inhibitors That Act by Covalently Bonding to Two Active Site Residues. J. Am. Chem. Soc. 2013, 135, 6289-6299.

(10) Palermo, G.; Branduardi, D.; Masetti, M.; Lodola, A.; Mor, M.; Piomelli, D.; Cavalli, A.; De Vivo, M. Covalent Inhibitors of Fatty Acid Amide Hydrolase: A Rationale for the Activity of Piperidine and Piperazine Aryl Ureas. J. Med. Chem. 2011, 54, 6612-6623.

(11) Piomelli, D. The Endocannabinoid System: A Drug Discovery Perspective. Curr. Opin. Investig. Drugs. 2005, 6, 672-679.

(12) Bracey, M. H.; Hanson, M. A.; Masuda, K. R.; Stevens, R. C.; Cravatt, B. F. Structural Adaptations in a Membrane Enzyme that Terminates Endocannabinoid Signaling. Science 2002, 298, 17931796.

(13) McKinney, M. K.; Cravatt, B. F. Structure and Function of Fatty Acid Amide Hydrolase. Annu. Rev. Biochem. 2005, 74, 411-432.

(14) Palermo, G.; Campomanes, P. R.; Neri, M.; Piomelli, D.; Cavalli, A.; Rothlisberger, U.; De Vivo, M. Wagging the Tail: Essential Role of Substrate Flexibility in FAAH Catalysis. J. Chem. Theory Comput. 2013, 9, 1202-1213.

(15) McKinney, M. K.; Cravatt, B. F. Evidence for Distinct Roles in Catalysis for Residues of the Serine-Serine-Lysine Catalytic Triad of Fatty Acid Amide Hydrolase. J. Biol. Chem. 2003, 278, 37393-37399.

(16) Mileni, M.; Kamtekar, S.; Wood, D. C.; Benson, T. E.; Cravatt, B. F.; Stevens, R. C. Crystal Structure of Fatty Acid Amide Hydrolase Bound to the Carbamate Inhibitor URB597: Discovery of a Deacylating Water Molecule and Insight into Enzyme Inactivation. J. Mol. Biol. 2010, 400, 743-754.

(17) Acevedo, O.; Jorgensen, W. L. Advances in Quantum and Molecular Mechanical (QM/MM) Simulations for Organic and Enzymatic Reactions. Acc. Chem. Res. 2010, 43, 142-151.

(18) van der Kamp, M. W.; Mulholland, A. J. Combined Quantum Mechanics/Molecular Mechanics (QM/MM) Methods in Computational Enzymology. Biochemistry 2013, 52, 2708-2728.

(19) Capoferri, L.; Mor, M.; Sirirak, J.; Chudyk, E.; Mulholland, A. J.; Lodola, A. Application of a SCC-DFTB QM/MM Approach to the Investigation of the Catalytic Mechanism of Fatty Acid Amide Hydrolase. J. Mol. Model. 2011, 17, 2375-2383.

(20) Chudyk, E. I.; Dyguda-Kazimierowicz, E.; Langner, K. M.; Sokalski, W. A.; Lodola, A.; Mor, M.; Sirirak, J.; Mulholland, A. J. Nonempirical Energetic Analysis of Reactivity and Covalent Inhibition of Fatty Acid Amide Hydrolase. J. Phys. Chem. B 2013, 117, 66566666.

(21) Lodola, A.; Capoferri, L.; Rivara, S.; Chudyk, E.; Sirirak, J.; Dyguda-Kazimierowicz, E.; Andrzej Sokalski, W.; Mileni, M.; Tarzia, G.; Piomelli, D.; Mor, M.; Mulholland, A. J. Understanding the Role of Carbamate Reactivity in Fatty Acid Amide Hydrolase Inhibition by QM/MM Mechanistic Modelling. Chem. Commun. (Cambridge, U. K.) 2011, 47, 2517-2519.
(22) Lodola, A.; Capoferri, L.; Rivara, S.; Tarzia, G.; Piomelli, D.; Mulholland, A.; Mor, M. Quantum Mechanics/Molecular Mechanics Modeling of Fatty Acid Amide Hydrolase Reactivation Distinguishes Substrate from Irreversible Covalent Inhibitors. J. Med. Chem. 2013, $56,2500-2512$.

(23) Lodola, A.; Mor, M.; Hermann, J. C.; Tarzia, G.; Piomelli, D.; Mulholland, A. J. QM/MM Modelling of Oleamide Hydrolysis in Fatty Acid Amide Hydrolase (FAAH) Reveals a New Mechanism of Nucleophile Activation. Chem. Commun. (Cambridge, U. K.) 2005, 4399-4401.

(24) Lodola, A.; Mor, M.; Rivara, S.; Christov, C.; Tarzia, G.; Piomelli, D.; Mulholland, A. J. Identification of Productive Inhibitor Binding Orientation in Fatty Acid Amide Hydrolase (FAAH) by QM/ MM Mechanistic Modelling. Chem. Commun. (Cambridge, U. K.) 2008, 214-416.

(25) Lodola, A.; Mor, M.; Sirirak, J.; Mulholland, A. J. Insights into the Mechanism and Inhibition of Fatty Acid Amide Hydrolase from Quantum Mechanics/Molecular Mechanics (QM/MM) Modelling. Biochem. Soc. Trans. 2009, 37, 363-367.

(26) Lodola, A.; Mor, M.; Zurek, J.; Tarzia, G.; Piomelli, D.; Harvey, J. N.; Mulholland, A. J. Conformational Effects in Enzyme Catalysis: Reaction via a High Energy Conformation in Fatty Acid Amide Hydrolase. Biophys. J. 2006, 15, L20-L22.

(27) Lodola, A.; Sirirak, J.; Fey, N.; Rivara, S.; Mor, M.; Mulholland, A. J. Structural Fluctuations in Enzyme-Catalyzed Reactions: Determinants of Reactivity in Fatty Acid Amide Hydrolase from Multivariate Statistical Analysis of Quantum Mechanics/Molecular Mechanics Paths. J. Chem. Theory Comput. 2010, 6, 2948-2960.

(28) Boger, D. L.; Miyauchi, H.; Du, W.; Hardouin, C.; Fecik, R. A.; Cheng, H.; Hwang, I.; Hedrick, M. P.; Leung, D.; Acevedo, O.; Guimaraes, C. R.; Jorgensen, W. L.; Cravatt, B. F. Discovery of a Potent, Selective, and Efficacious Class of Reversible AlphaKetoheterocycle Inhibitors of Fatty Acid Amide Hydrolase Effective as Analgesics. J. Med. Chem. 2005, 48, 1849-1856.

(29) Guimaraes, C. R.; Boger, D. L.; Jorgensen, W. L. Elucidation of Fatty Acid Amide Hydrolase Inhibition by Potent Alpha-Ketoheterocycle Derivatives from Monte Carlo Simulations. J. Am. Chem. Soc. 2005, 127, 17377-17784.

(30) Tubert-Brohman, I.; Acevedo, O.; Jorgensen, W. L. Elucidation of Hydrolysis Mechanisms for Fatty Acid Amide Hydrolase and its Lys142Ala Variant via QM/MM Simulations. J. Am. Chem. Soc. 2006, 128, 16904-16913.

(31) Warshel, A.; Levitt, M. Theoretical Studies of Enzymic Reactions: Dielectric, Electrostatic and Steric Stabilization of the Carbonium Ion in the Reaction of Lysozyme. J. Mol. Biol. 1976, 103, 227-249.

(32) Syren, P. O. The Solution of Nitrogen Inversion in Amidases. FEBS J. 2013, 280, 3069-3083.

(33) Deslongchamps, P. Stereoelectronic Control in the Cleavage of Tetrahedral Intermediates in the Hydrolysis of Esters and Amides. Tetrahedron 1975, 31, 2463-2490.

(34) Kamerlin, S. C.; Chu, Z. T.; Warshel, A. On Catalytic Preorganization in Oxyanion Holes: Highlighting the Problems with the Gas-Phase Modeling of Oxyanion Holes and Illustrating the Need for Complete Enzyme Models. J. Org. Chem. 2010, 75, 6391-6401.

(35) Ahn, K.; Johnson, D. S.; Mileni, M.; Beidler, D.; Long, J. Z.; McKinney, M. K.; Weerapana, E.; Sadagopan, N.; Liimatta, M.; Smith, S. E.; Lazerwith, S.; Stiff, C.; Kamtekar, S.; Bhattacharya, K.; Zhang, Y.; Swaney, S.; Van Becelaere, K.; Stevens, R. C.; Cravatt, B. F. Discovery and Characterization of a Highly Selective FAAH Inhibitor that Reduces Inflammatory Pain. Chem. Biol. 2009, 16, 411-420.

(36) Dive, G.; Dehareng, D. Serine Peptidase Catalytic Machinery: Cooperative One-Step Mechanism. Int. J. Quantum Chem. 1999, 73, $161-174$.

(37) Strynadka, N. C. J.; Adachi, H.; Jensen, S. E.; Johns, K.; Sielecki, A.; Betzel, C.; Sutoh, K.; James, M. N. G. Molecular-Structure of the Acyl-Enzyme Intermediate in Beta-Lactam Hydrolysis at 1.7 Angstrom Resolution. Nature 1992, 359, 700-705. 
(38) Jorgensen, W. L.; Chandrasekhar, J.; Madura, J. D.; Impey, R. W.; Klein, M. L. Comparison of Simple Potential Functions for Simulating Liquid Water. J. Chem. Phys. 1983, 79, 926-935.

(39) Car, R.; Parrinello, M. Unified Approach for Molecular Dynamics and Density-Functional Theory. Phys. Rev. Lett. 1985, 55, 2471-2474.

(40) Cornell, W. D.; Cieplak, P.; Bayly, C. I.; Gould, I. R.; Merz, K. M.; Ferguson, D. M.; Spellmeyer, D. C.; Fox, T.; Caldwell, J. W.; Kollman, P. A. A Second Generation Force Field for the Simulation of Proteins, Nucleic Acids, and Organic Molecules. J. Am. Chem. Soc. 1995, 117, 5179-5197.

(41) Wang, J.; Wolf, R. M.; Caldwell, J. W.; Kollman, P. A.; Case, D. A. Development and Testing of a General Amber Force Field. J. Comput. Chem. 2004, 25, 1157-1174.

(42) Bayly, C. I.; Cieplak, P.; Cornell, W. D.; Kollman, P. A. A WellBehaved Electrostatic Potential Based Method Using Charge Restraints for Deriving Atomic Charges: The RESP Model. J. Phys. Chem. 1993, 97, 10269-10280.

(43) Hess, G.; Bekker, H.; Berendsen, H. J. C.; Fraaije, J. G. E. M. Lincs: A Linear Constraint Solver for Molecular Simulations. J. Comput. Chem. 1997, 18, 1463-1472.

(44) Van der Spoel, D.; Lindahl, E.; Hess, B.; Groenhof, G.; Mark, A. E.; Berendsen, H. J. C. Gromacs: Fast, Flexible and Free. J. Comput. Chem. 2005, 26, 1701-1718.

(45) Laio, A.; VandeVondele, J.; Rothlisberger, U. A Hamiltonian Electrostatic Coupling Scheme for Hybrid Car-Parrinello Molecular Dynamics Simulations. J. Chem. Phys. 2002, 116, 6941-6947.

(46) Parrinello, M.; Andreoni, W.; Curioni, A. CPMD; I.B.M. Corporation and Max-Planck Institut: Armonk, New York, and Stuttgart, Germany, 2000.

(47) Becke, A. D. Density-Functional Exchange-Energy Approximation with Correct Asymptotic-Behavior. Phys. Rev. A 1988, 38, 3098-3100.

(48) Lee, C. T.; Yang, W. T.; Parr, R. G. Development of the ColleSalvetti Correlation-Energy Formula into a Functional of the ElectronDensity. Phys. Rev. B 1988, 37, 785-789.

(49) Carloni, P.; Rothlisberger, U.; Parrinello, M. The Role and Perspective of $\mathrm{Ab}$ Initio Molecular Dynamics in the Study of Biological Systems. Acc. Chem. Res. 2002, 35, 455-464.

(50) De Vivo, M.; Cavalli, A.; Carloni, P.; Recanatini, M. Computational Study of the Phosphoryl Transfer Catalyzed by a Cyclin-Dependent Kinase. Chemistry 2007, 13, 8437-8444.

(51) De Vivo, M.; Dal Peraro, M.; Klein, M. L. Phosphodiester Cleavage in Ribonuclease $\mathrm{H}$ Occurs via an Associative Two-MetalAided Catalytic Mechanism. J. Am. Chem. Soc. 2008, 130, 1095510962.

(52) von Lilienfeld, O. A.; Tavernelli, I.; Rothlisberger, U.; Sebastiani, D. Variational Optimization of Effective Atom Centered Potentials for Molecular Properties. J. Chem. Phys. 2005, 122, 14113.

(53) Troullier, N.; Martins, J. L. Efficient Pseudopotentials for PlaneWave Calculations. II. Operators for Fast Iterative Diagonalization. Phys. Rev. B.: Condens. Matter Mater. Phys. 1991, 43, 8861-8869.

(54) Hockney, R. W. Methods Comput. Phys. 1970, 9, 135.

(55) Biarnes, X.; Ardevol, A.; Iglesias-Fernandez, J.; Planas, A.; Rovira, C. Catalytic Itinerary in 1,3-1,4-beta-Glucanase Unraveled by QM/MM Metadynamics. Charge Is Not Yet Fully Developed at the Oxocarbenium Ion-like Transition State. J. Am. Chem. Soc. 2011, 133, 20301-20309.

(56) Brunk, E.; Ashari, N.; Athri, P.; Campomanes, P.; de Carvalho, F. F.; Curchod, B. F. E.; Diamantis, P.; Doemer, M.; Garrec, J.; Laktionov, A.; Micciarelli, M.; Neri, M.; Palermo, G.; Penfold, T. J.; Vanni, S.; Tavernelli, I.; Rothlisberger, U. Pushing the Frontiers of First-Principles Based Computer Simulations of Chemical and Biological Systems. Chimia 2011, 65, 667-671.

(57) De Vivo, M.; Ensing, B.; Dal Peraro, M.; Gomez, G. A.; Christianson, D. W.; Klein, M. L. Proton Shuttles and Phosphatase Activity in Soluble Epoxide Hydrolase. J. Am. Chem. Soc. 2007, 129, 387-394.
(58) Palermo, G.; Stenta, M.; Cavalli, A.; Dal Peraro, M.; De Vivo, M. Molecular Simulations Highlight the Role of Metals in Catalysis and Inhibition of Type II Topoisomerase. J. Chem. Theory Comput. 2013, 9, 857-862.

(59) Hoover, W. G. Canonical Dynamics - Equilibrium Phase-Space Distributions. Phys. Rev. A 1985, 31, 1695-1697.

(60) Nose, S. An Extension of the Canonical Ensemble MolecularDynamics Method. Mol. Phys. 1986, 57, 187-191.

(61) Mileni, M.; Johnson, D. S.; Wang, Z.; Everdeen, D. S.; Liimatta, M.; Pabst, B.; Bhattacharya, K.; Nugent, R. A.; Kamtekar, S.; Cravatt, B. F.; Ahn, K.; Stevens, R. C. Structure-Guided Inhibitor Design for Human FAAH by Interspecies Active Site Conversion. Proc. Natl. Acad. Sci. U. S. A. 2008, 105, 12820-12824.

(62) Seierstad, M.; Breitenbucher, J. G. Discovery and Development of Fatty Acid Amide Hydrolase (FAAH) Inhibitors. J. Med. Chem. 2008, 51, 7327-7343.

(63) Garcia-Viloca, M.; Gao, J.; Karplus, M.; Truhlar, D. G. How Enzymes Work: Analysis by Modern Rate Theory and Computer Simulations. Science 2004, 303, 186-195.

(64) Masgrau, L.; Roujeinikova, A.; Johannissen, L. O.; Hothi, P.; Basran, J.; Ranaghan, K. E.; Mulholland, A. J.; Sutcliffe, M. J.; Scrutton, N. S.; Leys, D. Atomic Description of an Enzyme Reaction Dominated by Proton Tunneling. Science 2006, 312, 237-241.

(65) Mulholland, A. J. Chemical Accuracy in QM/MM Calculations on Enzyme-Catalysed Reactions. Chem. Cent. J. 2007, 1.

(66) Becke, A. D. A New Mixing of Hartree-Fock and Local Density-Functional Theories. J. Chem. Phys. 1993, 98, 1372-1377.

(67) Kim, K.; Jordan, K. D. Comparison of Density Functional and MP2 Calculations on the Water Monomer and Dimer. J. Phys. Chem. 1994, 98, 10089-10094.

(68) Stephens, P. J.; Devlin, C. F.; Chabalowski, C. F.; Frisch, M. J. $\mathrm{Ab}$ Initio Calculation of Vibrational Absorption and Circular Dichroism Spectra Using Density Functional Force Fields. J. Phys. Chem. 1994, 98, 11623-11627.

(69) Boger, D. L.; Fecik, R. A.; Patterson, J. E.; Miyauchi, H.; Patricelli, M. P.; Cravatt, B. F. Fatty Acid Amide Hydrolase Substrate Specificity. Bioorg. Med. Chem. Lett. 2000, 10, 2613-2616.

(70) Greenberg, A.; Breneman, C. M.; Liebman, J. F. The Amide Linkage: Structural Significance in Chemistry, Biochemistry, and Materials Science; Wiley: Hoboken, NJ, 2000.

(71) Ahn, K.; Johnson, D. S.; Fitzgerald, L. R.; Liimatta, M.; Arendse, A.; Stevenson, T.; Lund, E. T.; Nugent, R. A.; Nomanbhoy, T. K.; Alexander, J. P.; Cravatt, B. F. Novel Mechanistic Class of Fatty Acid Amide Hydrolase Inhibitors with Remarkable Selectivity. Biochemistry 2007, 46, 13019-13030.

(72) Labahn, J.; Neumann, S.; Buldt, G.; Kula, M. R.; Granzin, J. An Alternative Mechanism for Amidase Signature Enzymes. J. Mol. Biol. 2002, 322, 1053-1064.

(73) Shin, S.; Yun, Y. S.; Koo, H. M.; Kim, Y. S.; Choi, K. Y.; Oh, B. H. Characterization of a Novel Ser-cisSer-Lys Catalytic Triad in Comparison with the Classical Ser-His-Asp Triad. J. Biol. Chem. 2003, 278, 24937-24943.

(74) Lu, D. S.; Futterer, K.; Korolev, S.; Zheng, X. L.; Tan, K.; Waksman, G.; Sadler, J. E. Crystal Structure of Enteropeptidase Light Chain Complexed with an Analog of the Trypsinogen Activation Peptide. J. Mol. Biol. 1999, 292, 361-373.

(75) Scheidig, A. J.; Hynes, T. R.; Pelletier, L. A.; Wells, J. A.; Kossiakoff, A. A. Crystal Structures of Bovine Chymotrypsin and Trypsin Complexed to the Inhibitor Domain of Alzheimer's Amyloid Beta-Protein Precursor (APPI) and Basic Pancreatic Trypsin Inhibitor (BPTI): Engineering of Inhibitors with Altered Specificities. Protein Sci. 1997, 6, 1806-1824.

(76) Fulop, V.; Szeltner, Z.; Renner, V.; Polgar, L. Structures of Prolyl Oligopeptidase Substrate/Inhibitor Complexes - Use of Inhibitor Binding for Titration of the Catalytic Histidine Residue. J. Biol. Chem. 2001, 276, 1262-1266.

(77) McLuskey, K.; Paterson, N. G.; Bland, N. D.; Isaacs, N. W.; Mottram, J. C. Crystal Structure of Leishmania major Oligopeptidase 
B Gives Insight into the Enzymatic Properties of a Trypanosomatid Virulence Factor. J. Biol. Chem. 2010, 285, 39249-39259.

(78) Fujinaga, M.; Cherney, M. M.; Tarasova, N. I.; Bartlett, P. A.; Hanson, J. E.; James, M. N. G. Structural Study of the Complex Between Human Pepsin and a Phosphorus-Containing Peptidic Transition-State Analog. Acta Crystallogr. D 2000, 56, 272-279.

(79) Schroder, E.; Phillips, C.; Garman, E.; Harlos, K.; Crawford, C. $\mathrm{X}$-ray Crystallographic Structure of a Papain Leupeptin Complex. FEBS Lett. 1993, 315, 38-42.

(80) Lodola, A.; Branduardi, D.; De Vivo, M.; Capoferri, L.; Mor, M.; Piomelli, D.; Cavalli, A. A Catalytic Mechanism for Cysteine Nterminal Nucleophile Hydrolases, as Revealed by Free Energy Simulations. PLoS One 2012, 7, e32397.

(81) Stennicke, H. R.; Mortensen, U. H.; Breddam, K. Studies on the Hydrolytic Properties of (Serine) Carboxypeptidase Y. Biochemistry 1996, 35, 7131-7141.

(82) Pearl, L. H. The Catalytic Mechanism of Aspartic Proteinases.

FEBS Lett. 1987, 214, 8-12.

(83) Campestre, C.; Agamennone, M.; Tortorella, P.; Preziuso, S.; Biasone, A.; Gavuzzo, E.; Pochetti, G.; Mazza, F.; Hiller, O.; Tschesche, H.; Consalvi, V.; Gallina, C. N-Hydroxyurea as Zinc Binding Group in Matrix Metalloproteinase Inhibition: Mode of Binding in a Complex with MMP-8. Bioorg. Med. Chem. Lett. 2006, 16, 20-24.

(84) Fleming, C. D.; Edwards, C. C.; Kirby, S. D.; Maxwell, D. M.; Potter, P. M.; Cerasoli, D. M.; Redinbo, M. R. Crystal Structures of Human Carboxylesterase 1 in Covalent Complexes with the Chemical Warfare Agents Soman and Tabun. Biochemistry 2007, 46, 5063-5071.

(85) Uppenberg, J.; Ohrner, N.; Norin, M.; Hult, K.; Kleywegt, G. J.; Patkar, S.; Waagen, V.; Anthonsen, T.; Jones, T. A. Crystallographic and Molecular-Modeling Studies of Lipase B from Candida Antarctica Reveal a Stereospecificity Pocket for Secondary Alcohols. Biochemistry 1995, 34, 16838-16851.

(86) Syren, P. O.; Hult, K. Amidases Have a Hydrogen Bond that Facilitates Nitrogen Inversion, but Esterases Have Not. Chem CatChem 2011, 3, 853-860.

(87) Yasuhira, K.; Shibata, N.; Mongami, G.; Uedo, Y.; Atsumi, Y.; Kawashima, Y.; Hibino, A.; Tanaka, Y.; Lee, Y. H.; Kato, D.; Takeo, M.; Higuchi, Y.; Negoro, S. X-ray Crystallographic Analysis of the 6Aminohexanoate Cyclic Dimer Hydrolase Catalytic Mechanism and Evolution of an Enzyme Responsible for Nylon-6 Byproduct Degradation. J. Biol. Chem. 2010, 285, 1239-1248.

(88) Kim, H.; Lipscomb, W. N. Comparison of the Structures of Three Carboxypeptidase A-Phosphonate Complexes Determined by X-ray Crystallography. Biochemistry 1991, 30, 8171-80.

(89) Klusak, V.; Barinka, C.; Plechanovova, A.; Mlcochova, P.; Konvalinka, J.; Rulisek, L.; Lubkowski, J. Reaction Mechanism of Glutamate Carboxypeptidase II Revealed by Mutagenesis, X-ray Crystallography, and Computational Methods. Biochemistry 2009, 48, 4126-4138.

(90) McVey, C. E.; Walsh, M. A.; Dodson, G. G.; Wilson, K. S.; Brannigan, J. A. Crystal Structures of Penicillin Acylase EnzymeSubstrate Complexes: Structural Insights into the Catalytic Mechanism. J. Mol. Biol. 2001, 313, 139-150.

(91) Rossocha, M.; Schultz-Heienbrok, R.; von Moeller, H.; Coleman, J. P.; Saenger, W. Conjugated Bile Acid Hydrolase is a Tetrameric N-Terminal Thiol Hydrolase with Specific Recognition of its Cholyl but not of its Tauryl Product. Biochemistry 2005, 44, 57395748.

(92) Shin, S.; Yun, Y. S.; Koo, H. M.; Kim, Y. S.; Choi, K. Y.; Oh, B. $\mathrm{H}$. Characterization of a Novel Ser-cisSer-Lys Catalytic Triad in Comparison with the Classical Ser-His-Asp Triad. J. Biol. Chem. 2003, 278, 24937-24943.

(93) Derewenda, U.; Brzozowski, A. M.; Lawson, D. M.; Derewenda, Z. S. Catalysis at the Interface: The Anatomy of a Conformational Change in a Triglyceride Lipase. Biochemistry 1992, 31, 1532-1541.

(94) Kiss, G.; Celebi-Olcum, N.; Moretti, R.; Baker, D.; Houk, K. N. Computational Enzyme Design. Angew. Chem., Int. Ed. Engl. 2013, 52, $5700-5725$.
(95) De Vivo, M. Bridging Quantum Mechanics and Structure-Based Drug Design. Frontiers in Bioscience 2011, 16, 1619-1633.

(96) Lodola, A.; De Vivo, M. The Increasing Role of QM/MM in Drug Discovery. In Structural and Mechanistic Enzymology: Bringing Together Experiments and Computing; Christov, C., KarabenchevaChristova, T., Eds.; Elsevier: Oxford, 2012.

(97) Krakowiak, A.; Pace, H. C.; Blackburn, G. M.; Adams, M.; Mekhalfia, A.; Kaczmarek, R.; Baraniak, J.; Stec, W. J.; Brenner, C. Biochemical, Crystallographic, and Mutagenic Characterization of Hint, the AMP-Lysine Hydrolase, with Novel Substrates and Inhibitors. J. Biol. Chem. 2004, 279, 18711-18716.

(98) Radisky, E. S.; Kwan, G.; Lu, C. J. K.; Koshland, D. E. Binding, Proteolytic, and Crystallographic Analyses of Mutations at the Protease-Inhibitor Interface of the Subtilisin $\mathrm{BPN}^{\prime} /$ Chymotrypsin Inhibitor 2 Complex. Biochemistry 2004, 43, 13648-13656. 